\title{
Robust fault detection based on adaptive threshold generation using interval LPV observers
}

\author{
Saúl Montes de Oca, Vicenç Puig*,† and Joaquim Blesa \\ Advanced Control Systems (SAC) and Institut de Robòtica i Informàtica Industrial (CSIC), \\ Universitat Politècnica de Catalunya, Pau Gargallo, 5, 08028 Barcelona, Spain
}

\begin{abstract}
SUMMARY
In this paper, robust fault detection based on adaptive threshold generation of a non-linear system described by means of a linear parameter-varying (LPV) model is addressed. Adaptive threshold is generated using an interval LPV observer that generates a band of predicted outputs taking into account the parameter uncertainties bounded using intervals. An algorithm that propagates the uncertainty based on zonotopes is proposed. The design procedure of this interval LPV observer is implemented via pole placement using linear matrix inequalities. Finally, the minimum detectable fault is characterized using fault sensitivity analysis and residual uncertainty bounds. Two examples, one based on a quadruple-tank system and another based on a two-degree of freedom helicopter, are used to assess the validity of the proposed fault detection approach.
\end{abstract}

KEY WORDS: fault detection; linear parameter-varying; interval LPV observer; linear matrix inequalities; zonotopes; minimum detectable fault

\section{INTRODUCTION}

Many model-based fault detection techniques, mostly based on linear models, have been investigated and developed in the literature over the last few years [1-3]. However, physical systems are inherently non-linear. This has motivated the interest of researchers in the development and application of non-linear FDI methodologies [4]. An attractive alternative to represent non-linear systems is to use linear parameter-varying (LPV) techniques (see $[4,5])$. The LPV approach is particularly appealing whenever non-linear plants can be modeled as time-varying systems with online measurable state-depending parameters $[6,7]$.

But, in the LPV approach, as in any model-based FDI approach, modeling errors and disturbances are always presented when applied to real complex engineering systems. Hence, in order to increase the reliability and performance of model-based fault detection, the development of robust fault detection algorithms should be addressed. The robustness of a fault detection system means that it must only be sensitive to faults, even in the presence of model-reality differences [1]. One of the approaches to robustness, known as active, is based on generating residuals that are insensitive to uncertainty, while at the same time sensitive to faults. This approach has been extensively developed these last years for several researchers using different techniques: unknown input observers [8], eigenstructure assignment [9] or structured parity equations [2]. Extensions to LPV systems have recently started to appear and can be found in [4, 10-13]. Using one the active approaches, the robustness with respect to unknown disturbances is solved. But, the robustness

\footnotetext{
${ }^{*}$ Correspondence to: Vicenç Puig, Advanced Control Systems (SAC) and Institut de Robòtica i Informàtica IndustriaO

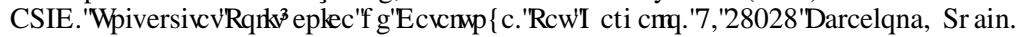

†E-mail: vicenc.puig@upc.edu
} 
problem with respect to modeling errors is more difficult to solve. The reason is that the uncertainty distribution matrix is normally unknown and time varying [2]. Moreover, there could be too many disturbances/uncertainties to be decoupled due to the lack of freedom. An alternative approach to handle modeling uncertainty, known as passive, is based on enhancing the robustness of the fault detection system at the decision-making stage. The aim with the passive approach is to determine, given a set of models, if there is any member in the set that can explain the measurements. A common approach to this problem is to propagate the model uncertainty to the alarm limits of the residuals generating an adaptive threshold. When the residuals are outside the alarm limits it is argued that model uncertainty alone cannot explain the residual; and therefore, a fault must have occurred. The goal of the adaptive threshold is to minimize the missing alarm rates due to the effects that modeling uncertainties will have on the residuals. However, this approach has the drawback that faults that produce a residual deviation smaller than the residual uncertainty due to parameter uncertainty will not be detected. Adaptive threshold generation has been a very active area of research in robust fault detection. Since the seminal works of [14,15], many researchers have analyzed how the effect of model uncertainty should be taken into account when determining the optimal threshold to be used in residual evaluation. In these last years, the research on passive robust fault detection methods has been an active area in the FDI community [16-19]. Most of these methods, known as set-membership, assume that the noise and/or parameters are bounded.

The aim of this paper is to develop a passive robust fault detection method for non-linear systems that can be represented using LPV models. The time-varying parameters of the LPV model can be adapted using some known scheduling function that can be tuned using the LPV identification. However, as a result of this identification process there is some modeling uncertainty in the LPV parameters that should be considered in the fault detection. The proposed approach uses an interval LPV observer to generate an adaptive threshold using a zonotope-based algorithm inspired by Alamo et al. [20] who takes into account parametric uncertainty. This approach is presented in [21] for LTI systems and here is extended to LPV systems. Interval observers, proposed originally by Gouzé [22], provide at every time instant an interval that bounds the effect of parameter uncertainty on system states/outputs.

The observer gain plays an important role because it determines the residual sensitivity to a fault and the associated adaptive threshold derived from the model uncertainty [1]. In this paper, the interval LPV observer gain is designed using linear matrix inequality (LMI) pole placement. However, this implies that the LPV model of the system should be approximated by a polytopic LPV model as proposed by Apkarian et al. [5]. The characterization of the minimum detectable fault is carried out using the residual fault sensitivity analysis.

Finally, two examples, one based on a quadruple-tank system [13,23] and another based on a two-degree of freedom helicopter [24,25], are used to assess the validity of the proposed fault detection approach.

The structure of this paper is organized as follows: interval LPV observers are introduced in Section 2. In Section 3, passive robust fault detection using interval LPV observers is addressed and a method to design the observer is proposed. Section 4 presents the zonotope approach to approximate the set of states/outputs estimated by the interval LPV observer. In Section 5, the minimum detectable fault is characterized using fault residual sensitivity. In Section 6, the four tanks system is used to illustrate the proposed approach. Section 7 presents a second example based on a two-degree of freedom helicopter that is used to demonstrate the effectiveness of the fault detection approach.

\section{INTERVAL LPV OBSERVERS}

\subsection{LPV representation}

Let us consider that the non-linear system to be monitored can be described by the following LPV representation:

$$
x(k+1)=A\left(\tilde{\vartheta}_{k}\right) x(k)+B\left(\tilde{\vartheta}_{k}\right) u_{0}(k)+F_{a}\left(\tilde{\vartheta}_{k}\right) f_{a}(k)
$$




$$
y(k)=C\left(\tilde{\vartheta}_{k}\right) x(k)+D\left(\tilde{\vartheta}_{k}\right) u_{0}(k)+F_{y}\left(\tilde{\vartheta}_{k}\right) f_{y}(k)
$$

where $u_{0}(k) \in \mathfrak{R}^{n_{u}}$ is the real system input, $y(k) \in \mathfrak{R}^{n_{y}}$ is the system output, $x(k) \in \mathfrak{R}^{n_{x}}$ is the state-space vector, $f_{a}(k) \in \Re^{n_{u}}$ and $f_{y}(k) \in \Re^{n_{y}}$ represent faults in the actuators and system output sensors, respectively. $\tilde{\vartheta}_{k}:=\tilde{\vartheta}(k)$ is the system vector of time-varying parameters of dimension $n_{\vartheta}$, which change with the operating point scheduled by some measured system variables $p_{k}$ $\left(p_{k}:=p(k)\right)$ and can be estimated using some scheduling function $\tilde{\vartheta}_{k}=\tilde{f}\left(p_{k}\right)$.

In this paper, the kind of LPV systems considered satisfies that the time-varying parameter vector $\tilde{\vartheta}_{k}$ can be bounded within a polytope. For this reason, they are known as polytopic LPV systems [5]. In particular, the state-space matrices range in a polytope of matrices defined by the convex hull of a finite number of matrices $N$. That is

$$
\begin{aligned}
& \left(\begin{array}{lll}
A\left(\tilde{\vartheta}_{k}\right) & B\left(\tilde{\vartheta}_{k}\right) & F_{a}\left(\tilde{\vartheta}_{k}\right) \\
C\left(\tilde{\vartheta}_{k}\right) & D\left(\tilde{\vartheta}_{k}\right) & F_{y}\left(\tilde{\vartheta}_{k}\right)
\end{array}\right) \in C o\left\{\left(\begin{array}{ccc}
A_{j}\left(\tilde{\vartheta}^{j}\right) & B_{j}\left(\tilde{\vartheta}^{j}\right) & F_{a, j}\left(\tilde{\vartheta}^{j}\right) \\
C_{j}\left(\tilde{\vartheta}^{j}\right) & D_{j}\left(\tilde{\vartheta}^{j}\right) & F_{y, j}\left(\tilde{\vartheta}^{j}\right)
\end{array}\right), j=1, \ldots, N\right\}
\end{aligned}
$$

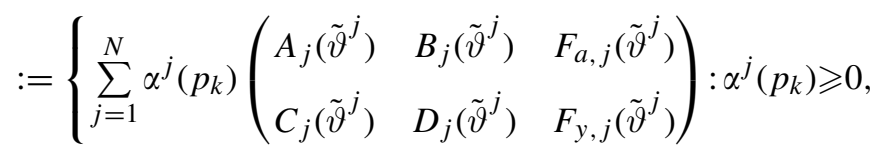

$$
\begin{aligned}
& \left.\sum_{j=1}^{N} \alpha^{j}\left(p_{k}\right)=1\right\}
\end{aligned}
$$

where $\tilde{\vartheta}^{j}$ is the vector of parameters corresponding to $j$ th model. Each $j$ th model is called a vertex system.

Consequently, the polytopic LPV model of the LPV system (1) and (2) can be expressed as follows:

$$
\begin{gathered}
x(k+1)=\sum_{j=1}^{N} \alpha^{j}\left(p_{k}\right)\left[A_{j}\left(\tilde{\vartheta}^{j}\right) x(k)+B_{j}\left(\tilde{\vartheta}^{j}\right) u_{0}(k)+F_{a, j}\left(\tilde{\vartheta}^{j}\right) f_{a}(k)\right] \\
y(k)=\sum_{j=1}^{N} \alpha^{j}\left(p_{k}\right)\left[C_{j}\left(\tilde{\vartheta}^{j}\right) x(k)+D_{j}\left(\tilde{\vartheta}^{j}\right) u_{0}(k)+F_{y, j}\left(\tilde{\vartheta}^{j}\right) f_{y}(k)\right]
\end{gathered}
$$

where $A_{j}, B_{j}, C_{j}$ and $D_{j}$ are the state-space matrices defined for $j$ th model. Notice that the state-space matrices of system (4) and (5) are equivalent to the interpolation between LTI models, that is: $A\left(\tilde{\vartheta}_{k}\right) \cong \sum_{j=1}^{N} \alpha^{j}\left(p_{k}\right) A_{j}\left(\tilde{\vartheta}^{j}\right)$ and analogously for $B\left(\tilde{\vartheta}_{k}\right), F_{a}\left(\tilde{\vartheta}_{k}\right), C\left(\tilde{\vartheta}_{k}\right), D\left(\tilde{\vartheta}_{k}\right)$ and $F_{y}\left(\tilde{\vartheta}_{k}\right)$. The polytopic system is scheduled through functions $\alpha^{j}\left(p_{k}\right)$, which lie in a convex set

$$
\Psi=\left\{\alpha^{j}\left(p_{k}\right) \in \Re^{N}, \alpha\left(p_{k}\right)=\left[\alpha^{1}\left(p_{k}\right), \ldots, \alpha^{N}\left(p_{k}\right)\right]^{\mathrm{T}}, \alpha^{j}\left(p_{k}\right) \geqslant 0, \sum_{j=1}^{N} \alpha^{j}\left(p_{k}\right)=1\right\}
$$

There are several ways of implementing (3) depending on how $\alpha^{j}\left(p_{k}\right)$ functions are defined [26]. Here, the function $\alpha^{j}\left(p_{k}\right)$ is defined via a barycentric combination of vertices as suggested by Apkarian et al. [5].

\subsection{Interval $L P V$ observer}

The system described by (1) and (2) is monitored using the following LPV observer with Luenberger structure assuming that we consider only strictly proper system, such that $D=0$ :

$$
\begin{gathered}
\hat{x}(k+1)=A_{0}\left(\vartheta_{k}\right) \hat{x}(k)+B\left(\vartheta_{k}\right) u(k)+L\left(\vartheta_{k}\right) y(k) \\
\hat{y}(k)=C\left(\vartheta_{k}\right) \hat{x}(k)
\end{gathered}
$$


where $A_{0}\left(\vartheta_{k}\right) \triangleq A\left(\vartheta_{k}\right)-L\left(\vartheta_{k}\right) C\left(\vartheta_{k}\right)$, which considers that the model parameters $\vartheta_{k}$ are estimated using an experimentally calibrated scheduling function $f\left(p_{k}\right)$ that is an approximation of $\tilde{f}\left(p_{k}\right) .^{\ddagger}$ The error introduced by this approximation is unknown but bounded, i.e. $\varepsilon_{k} \in[\underline{\varepsilon}, \bar{\varepsilon}]$. This error induces uncertainty about the exact knowledge of the real system parameters $\tilde{\vartheta}_{k}$

$$
\underline{\vartheta}_{k} \leqslant \tilde{\vartheta}_{k} \leqslant \bar{\vartheta}_{k}
$$

where $\underline{\vartheta}_{k}=f\left(p_{k}\right)-\underline{\varepsilon}$ and $\bar{\vartheta}_{k}=f\left(p_{k}\right)+\bar{\varepsilon}$.

In order to take into account this uncertainty in the fault detection using the LPV observer (7) and (8), its effect will be propagated to the estimated output and bounded by an interval. This is why this approach is known as an interval LPV observer. This interval LPV observer approach extends the one proposed in [29] for interval LTI models.

Using the polytopic approximation introduced in (3), the interval LPV observer (7) and (8) can also be approximated in a polytopic way as follows ${ }^{\S}$ :

$$
\begin{gathered}
\hat{x}(k+1)=\sum_{j=1}^{N} \alpha^{j}\left(p_{k}\right)\left[A_{0, j}\left(\vartheta^{j}\right) \hat{x}(k)+B_{j}\left(\vartheta^{j}\right) u(k)+L_{j}\left(\vartheta^{j}\right) y(k)\right] \\
\hat{y}(k)=\sum_{j=1}^{N} \alpha^{j}\left(p_{k}\right) C_{j}\left(\vartheta^{j}\right) \hat{x}(k)
\end{gathered}
$$

where $A_{0, j}\left(\vartheta^{j}\right) \triangleq A_{j}\left(\vartheta^{j}\right)-L_{j}\left(\vartheta^{j}\right) C_{j}\left(\vartheta^{j}\right)$ and $\vartheta^{j}$ are the estimated vector of the parameters corresponding to $j$ th model including the modeling uncertainties (9), i.e. $\vartheta^{j} \in\left[\underline{\vartheta}^{j}, \bar{\vartheta}^{j}\right] . u(k)$ is the measured system input vector, $\hat{x}(k)$ is the estimated system state vector, $\hat{y}(k)$ is the estimated system output vector and $L_{j}\left(\vartheta^{j}\right)$ is the observer gain that has to be designed in order to stabilize the observer given by (10) and (11) for all matrices in the polytope (3) and parameter uncertainty (9). "I The set of observer gains $L_{j}\left(\vartheta^{j}\right)$ will be interpolated to obtain the interval LPV observer gain as follows: $L\left(\vartheta_{k}\right)=\sum_{j=1}^{N} \alpha^{j}\left(p_{k}\right) L_{j}\left(\vartheta^{j}\right)$. Notice that the measured system input, $u(k)$, could be affected by the effect of faults in the input sensors, such that:

$$
u(k)=u_{0}(k)+F_{u}\left(\tilde{\vartheta}_{k}\right) f_{u}(k)
$$

where $u_{0}(k)$ is the real system input, $f_{u}(k) \in \mathfrak{R}^{n_{u}}$ is the input sensor fault, while $F_{u}\left(\tilde{\vartheta}_{k}\right) \in \Re^{n_{u} \times n_{u}}$ is its associated fault matrix. Each observer gain matrix $L_{j}\left(\vartheta^{j}\right) \in \Re^{n_{x} \times n_{y}}$ is designed to stabilize the corresponding $j$ th vertex and to guarantee a desired performance $\left(A_{0, j}\right)$ regarding fault detection [31].

\section{Definition 1}

Consider the interval LPV observer given by (10) and (11), the sequence of measured inputs $(u(i))_{0}^{k-1}$ and outputs $(y(i))_{0}^{k}$. It is assumed that the initial states are bounded by a known compact

\footnotetext{
† The scheduling function can be experimentally calibrated using the LPV identification algorithms as the ones proposed by Bamieh and Giarré [27] or Blesa et al. [28].

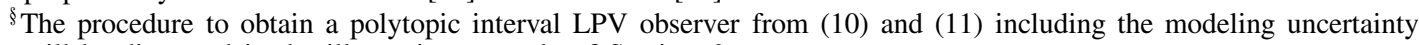
will be discussed in the illustrative example of Section 6.

IThe noise effect should also have been considered in the observer design and in the fault detection test (28) or (36). Although considering the noise effect in the detection test is straightforward if the noise is assumed bounded (as parameter uncertainty), this is not the case in the design of the LPV observer when considering noise and parameter uncertainty at the same time. The inclusion of parametric uncertainty in the LPV model is still a theoretical open issue [30].
} 
set $\mathbb{X}_{0}$. The exact uncertain estimated state set $\mathbb{X}_{k}$ at time $k$ is expressed by

$$
\begin{aligned}
\mathbb{X}_{k}= & \left\{\hat{x}_{k}:\left(\hat{x}(i)=\sum_{j=1}^{N} \alpha^{j}\left(p_{i}\right)\left[A_{0, j}\left(\vartheta^{j}\right) \hat{x}(i-1)+B_{j}\left(\vartheta^{j}\right) u(i-1)+L_{j}\left(\vartheta^{j}\right) y(i-1)\right]\right)_{i=1}^{k},\right. \\
& \left.\mid \hat{x}_{0} \in \mathbb{X}_{0}, \underline{\vartheta}^{j} \leqslant \vartheta^{j} \leqslant \bar{\vartheta}^{j}, j=1, \ldots, N\right\}
\end{aligned}
$$

where $A_{0, j}\left(\vartheta^{j}\right) \triangleq A_{j}\left(\vartheta^{j}\right)-L_{j}\left(\vartheta^{j}\right) C_{j}\left(\vartheta^{j}\right)$.

The uncertain state set described in Definition 1 at time $k$ can be computed approximately by admitting the rupture of the existing relations between variables of consecutive time instants. This allows to compute an approximation of this set from the approximate uncertain set at time $k-1$.

\section{Definition 2}

Consider the interval LPV observer given by (10) and (11), the set of uncertain states at time $k-1$ $\left(\mathbb{X}_{k-1}\right)$ and the input/output values $\left(u_{k-1}, y_{k-1}\right)$. Then, the approximated set of estimated states $\mathbb{X}_{k}^{e}$ at time $k$ based on the measurements up to time $k-1$ is defined as

$$
\begin{aligned}
\mathbb{X}_{k}^{e}= & \left\{\hat{x}_{k}: \hat{x}(k)=\sum_{j=1}^{N} \alpha^{j}\left(p_{k}\right)\left[A_{0, j}\left(\vartheta^{j}\right) \hat{x}(k-1)+B_{j}\left(\vartheta^{j}\right) u(k-1)+L_{j}\left(\vartheta^{j}\right) y(k-1)\right],\right. \\
& \left.\mid \hat{x}(k-1) \in \mathbb{X}_{k-1}, \underline{\vartheta}^{j} \leqslant \vartheta^{j} \leqslant \bar{\vartheta}^{j}, j=1, \ldots, N\right\}
\end{aligned}
$$

where $A_{0, j}\left(\vartheta^{j}\right) \triangleq A_{j}\left(\vartheta^{j}\right)-L_{j}\left(\vartheta^{j}\right) C_{j}\left(\vartheta^{j}\right)$.

Definitions 1 and 2 can be easily adapted to describe the exact uncertain estimated output set $\mathbb{Y}_{k}$ at time $k$ and the approximated set of estimated output $\mathbb{Y}_{k}^{e}$.

Since the exact set of estimated states $\mathbb{X}_{k}^{e}$ and outputs $\mathbb{Y}_{k}^{e}$ are difficult to compute, one way is to bound them using some geometric shapes easy to compute, for example: boxes (interval hull) as in [32], ellipsoids as in [33] or zonotopes as in [20].

Here, the set of estimated states $\mathbb{X}_{k}^{e}$ (or outputs $\mathbb{Y}_{k}^{e}$ ) introduced in Definition 2 will be approximated iteratively using zonotopes (see Section 4). From these zonotopes, an interval for each state variable can also be obtained by computing the interval hull of the zonotope. The sequence of interval hulls $\square \mathbb{X}_{k}^{e}$ and $\square \mathbb{Y}_{k}^{e}$ with $k \in[0, n]$ will be called the interval LPV observer estimation of the LPV system (10) and (11), where $n$ is the number of measurement data considered. Following the previous idea, Algorithm 1 is proposed to determine an approximation of set of uncertain estimated states and outputs (See Figure 1).

The implementation of steps 5-8 in the Algorithm 1 using zonotopes is described in detail in Section 4.

$$
\begin{array}{r}
\hat{x}(k+1)=\sum_{j=1}^{N} \alpha^{j}\left(p_{k}\right)\left[A_{0, j}\left(\vartheta^{j}\right) \hat{x}(k)+B_{j}\left(\vartheta^{j}\right) u(k)+L_{j}\left(\vartheta^{j}\right) y(k)\right] \\
\underset{k-1}{X_{k}^{e}} \stackrel{\text { Direct }}{\underset{\text { Image }}{\mathbf{X}}} \underset{\mathrm{X}_{k}^{e}}{\stackrel{\text { Direct }}{\mathbf{I m a g e}}} \mathrm{Y}_{k}^{e} \\
\hat{y}(k)=\sum_{j=1}^{N} \alpha^{j}\left(p_{k}\right)\left[C_{j}\left(\vartheta^{j}\right) \hat{x}(k)\right]
\end{array}
$$

Figure 1. Graphical interpretation of set-membership state estimation Algorithm 1. 


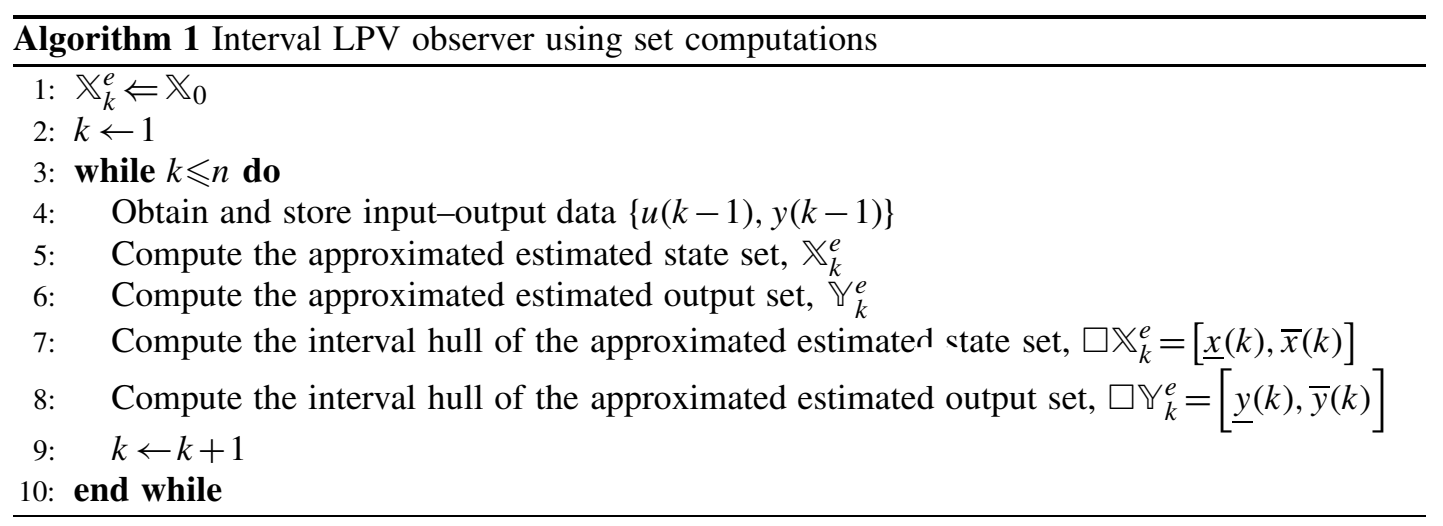

\subsection{Interval $L P V$ observer design}

In this paper, the interval LPV observer gain design (10) and (11) will be solved with the LMI pole placement technique $[11,31]^{\|}$that allows to locate the poles inside the unit circle using an LMI region taking into account the parameter variations and uncertainties.

According to Chilali et al. [31], an LMI region is any subset $\mathscr{D}$ of the complex plane that can be defined as

$$
\mathscr{D}=\left\{z \in C: L+z M+\bar{z} M^{\mathrm{T}}<0\right\}
$$

where $L$ and $M$ are the real matrices, such that $L^{\mathrm{T}}=L$. The matrix-valued function $f_{\mathscr{D}}(z)=$ $L+z M+\bar{z} M^{\mathrm{T}}$ is called the characteristic function. This LMI region $\mathscr{D}(-q, r)$ is characterized by a disk of radius $r$ and center $(-q, 0)$, such that the characteristic function is given by:

$$
f_{\mathscr{D}}(z)=\left[\begin{array}{cc}
-r & q+z \\
q+\bar{z} & -r
\end{array}\right]<0
$$

The design of the interval LPV observer (10) and (11), such that the observer poles are placed in the LMI region (16) requires to find for each vertex $j$ th (with $j \in[1, \ldots, N]$ ) the observer gain $L_{j}\left(\vartheta^{j}\right)$ and unknown symmetric matrix $X_{j}=X_{j}^{\mathrm{T}}>0$ that satisfies the following LMI:

$$
\left[\begin{array}{cc}
-r X_{j} & c X_{j}+\left(A_{0, j}\left(\vartheta^{j}\right)^{\mathrm{T}} X_{j}\right)^{\mathrm{T}} \\
\left(c+A_{0, j}\left(\vartheta^{j}\right)^{\mathrm{T}}\right) X_{j} & -r X_{j}
\end{array}\right]<0
$$

If $A_{0, j}\left(\vartheta^{j}\right)=A_{j}\left(\vartheta^{j}\right)-L_{j}\left(\vartheta^{j}\right) C_{j}\left(\vartheta^{j}\right)$, expression (17) is a bilinear matrix inequality (BMI), which cannot be solved with the classical LMI tools. But substituting $W_{j}=L_{j}\left(\vartheta^{j}\right)^{\mathrm{T}} X_{j}$ it is possible to transform it into:

$$
\left[\begin{array}{cc}
-r X_{j} & c X_{j}+X_{j}^{\mathrm{T}} A_{j}\left(\vartheta^{j}\right)-W_{j}^{\mathrm{T}} C_{j}\left(\vartheta^{j}\right) \\
\left(c+A_{j}\left(\vartheta^{j}\right)^{\mathrm{T}}\right) X_{j}-C_{j}\left(\vartheta^{j}\right)^{\mathrm{T}} W_{j} & -r X_{j}
\end{array}\right]<0
$$

Then, the design procedure boils down to solving the LMI (18) and then determining $L_{j}\left(\vartheta^{j}\right)=$ $\left(W_{j} X_{j}^{-1}\right)^{\mathrm{T}}$. Finally, the observer gains $L_{j}\left(\vartheta^{j}\right)$ will be interpolated to obtain the interval LPV observer gain as: $L\left(\vartheta_{k}\right)=\sum_{j=1}^{N} \alpha^{j}\left(p_{k}\right) L_{j}\left(\vartheta^{j}\right)$.

\footnotetext{
"Alternatively, the observer gain could have been designed to minimize the $H_{\infty}$ gain of the transfer function that relates the residual and the noise as suggested by Armeni et al. [13]. However, this approach does not consider parametric uncertainty being required an extension to be applied in the context of the interval LPV observers. This extension will be addressed in the future research.
} 


\section{FAULT DETECTION USING INTERVAL LPV OBSERVERS}

\subsection{Observer input/output form}

The system (1) and (2) can be expressed in input-output form using the shift operator** $q^{-1}$ and assuming zero initial conditions as follows ${ }^{\dagger \dagger}$ :

$$
y(k)=y_{0}(k)+G_{f_{a}}\left(q^{-1}, \tilde{\vartheta}_{k}\right) f_{a}(k)+G_{f_{y}}\left(q^{-1}, \tilde{\vartheta}_{k}\right) f_{y}(k)
$$

where

$$
\begin{gathered}
y_{0}(k)=G_{u}\left(q^{-1}, \tilde{\vartheta}_{k}\right) u_{0}(k) \\
G_{u}\left(q^{-1}, \tilde{\vartheta}_{k}\right)=C\left(\tilde{\vartheta}_{k}\right)\left(q I-A\left(\tilde{\vartheta}_{k}\right)\right)^{-1} B\left(\tilde{\vartheta}_{k}\right)+D\left(\tilde{\vartheta}_{k}\right) \\
G_{f_{a}}\left(q^{-1}, \tilde{\vartheta}_{k}\right)=C\left(\tilde{\vartheta}_{k}\right)\left(q I-A\left(\tilde{\vartheta}_{k}\right)\right)^{-1} F_{a}\left(\tilde{\vartheta}_{k}\right) \\
G_{f_{y}}\left(q^{-1}, \tilde{\vartheta}_{k}\right)=F_{y}\left(\tilde{\vartheta}_{k}\right)
\end{gathered}
$$

Alternatively, the observer described by Equations (10) and (11) can be expressed in input-output form by

$$
\begin{aligned}
\hat{y}(k) & =\sum_{j=1}^{N} \alpha^{j}\left(p_{k}\right)\left[G^{j}\left(q^{-1}, \vartheta^{j}\right) u(k)+H^{j}\left(q^{-1}, \vartheta^{j}\right) y(k)\right] \\
& =\sum_{j=1}^{N} \alpha^{j}\left(p_{k}\right)\left[G^{j}\left(q^{-1}, \vartheta^{j}\right) u_{0}(k)+H^{j}\left(q^{-1}, \vartheta^{j}\right) y(k)+G_{f_{u}}^{j}\left(q^{-1}, \vartheta^{j}\right) f_{u}(k)\right]
\end{aligned}
$$

where

$$
\begin{gathered}
G^{j}\left(q^{-1}, \vartheta^{j}\right)=C_{j}\left(\vartheta^{j}\right)\left(q I-A_{0, j}\left(\vartheta^{j}\right)\right)^{-1} B_{j}\left(\vartheta^{j}\right) \\
H^{j}\left(q^{-1}, \vartheta^{j}\right)=C_{j}\left(\vartheta^{j}\right)\left(q I-A_{0, j}\left(\vartheta^{j}\right)\right)^{-1} L_{j}\left(\vartheta^{j}\right) \\
G_{f_{u}}^{j}\left(q^{-1}, \vartheta^{j}\right)=G^{j}\left(q^{-1}, \vartheta^{j}\right) F_{u}\left(\vartheta^{j}\right)
\end{gathered}
$$

The effect of the uncertain parameters $\vartheta_{k}$ on the observer temporal response $\hat{y}\left(k, \vartheta_{k}\right)$ can be determined computing the estimated output set $\mathbb{Y}_{k}^{e}$ using Algorithm 1. The effect of the uncertainty in a particular output can be bounded using an interval

$$
\hat{y}(k) \in[\underline{\hat{y}}(k), \overline{\hat{y}}(k)]
$$

where

$$
\begin{aligned}
& \underline{\hat{y}}(k)=\min _{\underline{\vartheta}^{j} \leqslant \vartheta^{j} \leqslant \bar{\vartheta}^{j}, j=1, \ldots, N}\left\{\sum_{j=1}^{N} \alpha^{j}\left(p_{k}\right)\left[G^{j}\left(q^{-1}, \vartheta^{j}\right) u(k)+H^{j}\left(q^{-1}, \vartheta^{j}\right) y(k)\right]\right\} \\
& \overline{\hat{y}}(k)=\max _{\underline{\vartheta}^{j} \leqslant \vartheta^{j} \leqslant \bar{\vartheta}^{j}, j=1, \ldots, N}\left\{\sum_{j=1}^{N} \alpha^{j}\left(p_{k}\right)\left[G^{j}\left(q^{-1}, \vartheta^{j}\right) u(k)+H^{j}\left(q^{-1}, \vartheta^{j}\right) y(k)\right]\right\}
\end{aligned}
$$

This interval is computed independently for each output (neglecting couplings between outputs) through the interval hull of $\mathbb{Y}_{k}^{e}$, denoted as $\square \mathbb{Y}_{k}^{e}$, using Algorithm 1.

\footnotetext{
** The transfer functions of discrete models are usually written in terms of delay operator ' $q^{-1}$ '. This allows to reason with signals in time domain using the transfer function concept. The expression $G\left(q^{-1}\right)$ is named transfer operator and allows to describe models by difference equations with time-varying coefficients [34].

${ }^{\dagger}$ In the following, for simplicity and with abuse of notation, transfer functions are used for LPV systems, although computations are performed entirely using the state-space representation: $G \triangleq\left[\begin{array}{c|c}A & B \\ \hline C & 0\end{array}\right]$
} 


\subsection{Adaptive thresholding}

The application of interval LPV observers to fault detection consists of testing whether the measured output is consistent with the one given by an observer using a faultless model. If an inconsistency is detected, the existence of a fault is proved. This consistency check is based on generating a nominal residual comparing the measurements of physical variables $y(k)$ of the process with their estimation $\hat{y}(k)$ provided by the associated system model

$$
r(k)=y(k)-\hat{y}(k)
$$

where $r(k) \in \Re^{n_{y}}$ is the residual and $\hat{y}(k)$ is the prediction obtained using the nominal LPV model. According to Gertler [2], the computational form of the residual generator, obtained using (24), is

$$
r(k)=\sum_{j=1}^{N} \alpha^{j}\left(p_{k}\right)\left[-G^{j}\left(q^{-1}, \vartheta^{j}\right) u(k)+\left(I-H^{j}\left(q^{-1}, \vartheta^{j}\right)\right) y(k)\right]
$$

Alternatively, the residual given by (32) can be also expressed in terms of the effects caused by faults using its internal or unknown-input-effect form [2]. This form, obtained by combining (19), (24) and (31), is expressed as

$$
\begin{aligned}
r(k)= & r_{0}(k)+\sum_{j=1}^{N} \alpha^{j}\left(p_{k}\right)\left[\left(I-H^{j}\left(q^{-1}, \vartheta^{j}\right)\right)\left(G_{f y}^{j}\left(q^{-1}, \vartheta^{j}\right) f_{y}(k)+G_{f a}^{j}\left(q^{-1}, \vartheta^{j}\right) f_{a}(k)\right)\right. \\
& \left.-G_{f u}^{j}\left(q^{-1}, \vartheta^{j}\right) f_{u}(k)\right]
\end{aligned}
$$

where

$$
\begin{gathered}
\sum_{j=1}^{N} \alpha^{j}\left(p_{k}\right) G_{f y}^{j}\left(q^{-1}, \vartheta^{j}\right)=G_{f y}\left(q^{-1}, \tilde{\vartheta}_{k}\right), \quad \sum_{j=1}^{N} \alpha^{j}\left(p_{k}\right) G_{f a}^{j}\left(q^{-1}, \vartheta^{j}\right)=G_{f a}\left(q^{-1}, \tilde{\vartheta}_{k}\right) \\
r_{0}(k)=\sum_{j=1}^{N} \alpha^{j}\left(p_{k}\right)\left[-G^{j}\left(q^{-1}, \vartheta^{j}\right) u_{0}(k)+\left(I-H^{j}\left(q^{-1}, \vartheta^{j}\right)\right) y_{0}(k)\right]
\end{gathered}
$$

Notice that expression (35) represents the non-faulty residual. Comparing (32) and (35), it should be noticed that both $r_{0}(k)$ and $r(k)$ are affected in the same way by the observation gain $L$.

The residual generated by (31) will not be zero, even in a non-faulty scenario when considering model uncertainty located in parameters. To cope with the parameter uncertainty effect, a passive robust approach based on adaptive thresholding can be used [14]. Thus, using this passive approach, the effect of parameter uncertainty in the residual $r(k)$ (associated with each system output $y(k)$ ) is bounded by the interval

$$
r(k) \in[\underline{r}(k), \bar{r}(k)]
$$

where

$$
\underline{r}(k)=\underline{\hat{y}}(k)-\hat{y}(k) \quad \text { and } \quad \bar{r}(k)=\overline{\hat{y}}(k)-\hat{y}(k)
$$

$\hat{y}(k)$ is the nominal predicted output using interval LPV observer (10) and (11), $\hat{y}(k)$ and $\overline{\hat{y}}(k)$ are the bounds of the predicted output (28). The residual generated by (37) can be expressed in input-output form using the interval LPV observer (24) instead of interval LPV observer (10) and (11)

$$
\begin{aligned}
& \underline{r}(k)=\min _{\underline{\vartheta}^{j} \leqslant \vartheta^{j} \leqslant \bar{\vartheta}^{j}, j=1, \ldots, N}\left\{\sum_{j=1}^{N} \alpha^{j}\left(p_{k}\right)\left[\Delta G^{j}\left(q^{-1}, \vartheta^{j}\right) u(k)+\Delta H^{j}\left(q^{-1}, \vartheta^{j}\right) y(k)\right]\right\} \\
& \bar{r}(k)=\max _{\underline{\vartheta}^{j} \leqslant \vartheta^{j} \leqslant \bar{\vartheta}^{j}, j=1, \ldots, N}\left\{\sum_{j=1}^{N} \alpha^{j}\left(p_{k}\right)\left[\Delta G^{j}\left(q^{-1}, \vartheta^{j}\right) u(k)+\Delta H^{j}\left(q^{-1}, \vartheta^{j}\right) y(k)\right]\right\}
\end{aligned}
$$


where

$$
\begin{gathered}
\Delta G^{j}\left(q^{-1}, \vartheta^{j}\right)=G^{j}\left(q^{-1}, \vartheta^{j}\right)-G^{j}\left(q^{-1}, \vartheta_{0}^{j}\right) \\
\Delta H^{j}\left(q^{-1}, \vartheta^{j}\right)=H^{j}\left(q^{-1}, \vartheta^{j}\right)-H^{j}\left(q^{-1}, \vartheta_{0}^{j}\right)
\end{gathered}
$$

and $\vartheta_{0}^{j}$ are the nominal parameters.

Algorithm 2 implements the fault detection using interval LPV observers, the fault detection test is presented in (28). Analogously, the fault detection test (36) can be used.

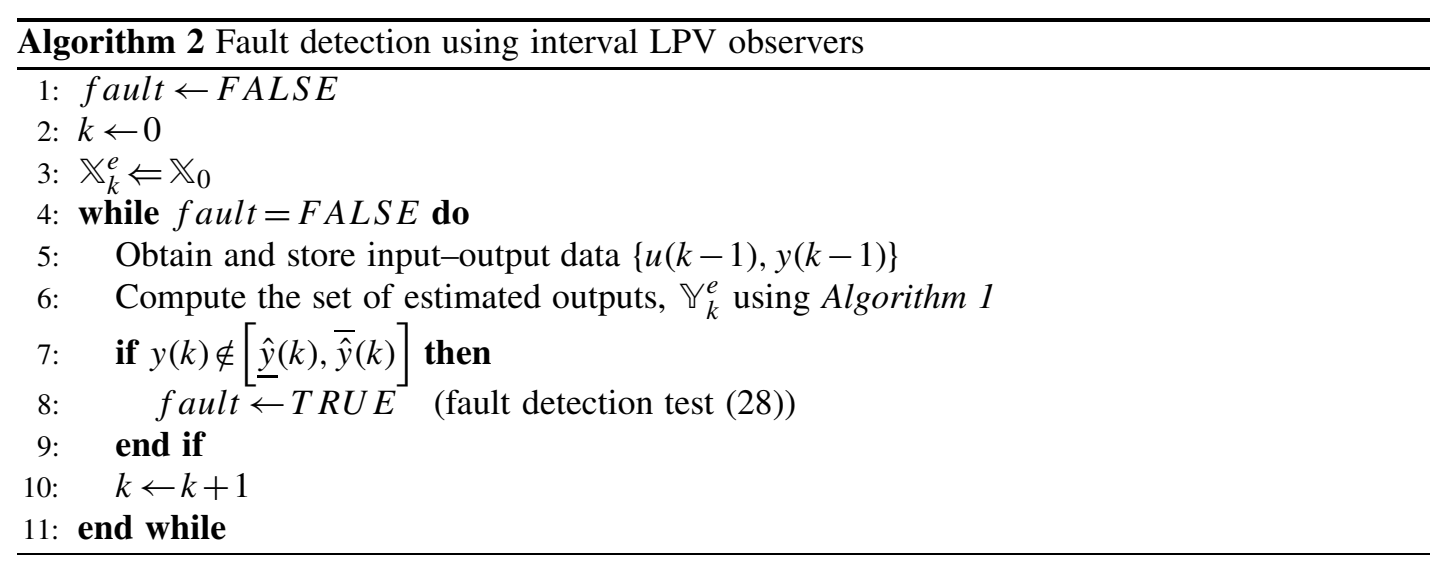

\section{IMPLEMENTATION OF INTERVAL LPV OBSERVERS USING ZONOTOPES}

\subsection{Introduction}

Here, zonotopes are used to bound the set of uncertain estimated states and outputs. Let us introduce zonotopes.

Definition 3

The Minkowski sum of two sets $\mathbb{X}$ and $\mathbb{Y}$ is defined by $\mathbb{X} \oplus \mathbb{Y}=\{x+y: x \in \mathbb{X}, y \in \mathbb{Y}\}$.

Definition 4

Given a center vector $\pi \in \Re^{n_{z}}$ and a matrix $H \in \Re^{n_{z} \times m_{z}}$, the Minkowski sum of the segments, defined by the columns of matrix $H$, is called a zonotope of order $m_{z}$ (see Figure 2). This set is represented as

$$
\mathbb{X}=\pi \oplus H \beta^{m_{z}}=\left\{\pi+H z: z \in \beta^{m_{z}}\right\}
$$

where $\beta^{m_{z}}$ is a unitary box, composed of $m_{z}$ unitary intervals.

\section{Definition 5}

The interval hull $\square \mathbb{X}$ of a closed set $\mathbb{X}$ is the smallest interval box that contains $\mathbb{X}$.

Given a zonotope $\mathbb{X}=\pi \oplus H \beta^{m_{z}}$, its interval hull can be easily computed by evaluating $\pi \oplus H \beta^{m_{z}}$ :

$$
\square \mathbb{X}=\left\{x \forall i=1, \ldots, n:\left|x_{i}-\pi_{i}\right| \leqslant\left\|H_{i}\right\|_{1}\right\}
$$

where $x_{i}$ and $\pi_{i}$ are the $i$ th components of $x$ and $\pi$, respectively, and $H_{i}$ is $i$ th-row of $H$. 


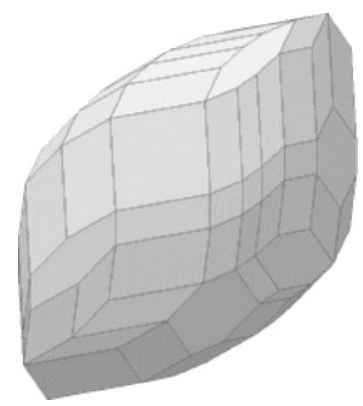

Figure 2. Zonotope of order $m_{z}=14$.

\subsection{Implementation of interval LPV observers using Zonotopes}

The steps 5 and 6 of the Algorithm 1 require computing the approximated state set $\mathbb{X}_{k}^{e}$ and output set $\mathbb{Y}_{k}^{e}$, respectively. These sets can be obtained using zonotopes by formulating the interval LPV observer (10) and (11) as follows":

$$
\begin{gathered}
\hat{x}(k+1)=\sum_{j=1}^{N} \alpha^{j}\left(p_{k}\right)\left[A_{0, j}\left(\vartheta^{j}\right) \hat{x}(k)+B_{0, j}\left(\vartheta^{j}\right) v(k)\right] \\
\hat{y}(k)=\sum_{j=1}^{N} \alpha^{j}\left(p_{k}\right) C_{j}\left(\vartheta^{j}\right) \hat{x}(k)
\end{gathered}
$$

where $A_{0, j}\left(\vartheta^{j}\right)=A_{j}\left(\vartheta^{j}\right)-L_{j}\left(\vartheta^{j}\right) C_{j}\left(\vartheta^{j}\right), B_{0, j}\left(\vartheta^{j}\right)=\left[B_{j}\left(\vartheta^{j}\right) L_{j}\left(\vartheta^{j}\right)\right]$ and $v(k)=[u(k) y(k)]^{\mathrm{T}}$, and using the following result:

Theorem 1 ('Zonotope Inclusion' (see Alamo et al. [20]))

Consider a family of zonotopes represented by $\mathbb{X}=\pi \oplus M \beta^{m_{z}}$, where $\pi \in \mathfrak{R}^{n_{z}}$ is a real vector and $M \in \Im^{n_{z} \times m_{z}}$ is an interval matrix. A zonotope inclusion $\diamond(\mathbb{X})$ is defined by

$$
\diamond(\mathbb{X})=\pi \oplus[\operatorname{mid}(M) G]\left[\begin{array}{l}
\beta^{m_{z}} \\
\beta^{n_{z}}
\end{array}\right]=\pi \oplus J \beta^{n_{z}+m_{z}}
$$

where $G \in \Re^{n_{z} \times n_{z}}$ is a diagonal matrix that satisfies: $G_{i i}=\sum_{j=1}^{m_{z}}\left(\operatorname{diam}\left(M_{i j}\right) / 2\right), i=1,2, \ldots, n$, 'mid' denotes the center and 'diam' the diameter of the interval according to Moore [35]. Under this definition, $\mathbb{X} \subseteq \diamond(\mathbb{X})$.

Note that this result is the generalization of the linear image of a zonotope that can be computed the following property:

Property 1 ('Zonotope Linear Image Transformation' (see Combastel [36])).

Consider a zonotope represented by $\mathbb{X}=\pi \oplus H \beta^{m_{z}}$, where $\pi \in \Re^{n_{z}}$ is a vector and $H \in \mathfrak{R}^{n_{z} \times m_{z}}$ is a matrix. The image of the zonotope $\mathbb{X}$ through a linear transformation $T \in \Re^{n_{z} \times n_{z}}$ is a zonotope $\mathbb{Y}$ defined by

$$
\mathbb{Y}=q_{z} \oplus N_{z} \beta^{m_{z}}
$$

\footnotetext{
$\$$ The computation using (41) and (42) requires $N$ interval observer simulations in parallel. When $N$ is large, the computation complexity could be important for some practical systems with fast dynamics. In this case, the exact LPV model (1) and (2) can be alternatively used by reformulating (41) and (42) as follows:

$$
\begin{gathered}
\hat{x}(k+1)=A_{0}\left(\vartheta_{k}\right) \hat{x}(k)+B_{0}\left(\vartheta_{k}\right) v(k) \\
\hat{y}(k)=C\left(\vartheta_{k}\right) \hat{x}(k)
\end{gathered}
$$

where $A_{0}\left(\vartheta_{k}\right)=A\left(\vartheta_{k}\right)-L\left(\vartheta_{k}\right) C\left(\vartheta_{k}\right), B_{0}\left(\vartheta_{k}\right)=\left[B\left(\vartheta_{k}\right) L\left(\vartheta_{k}\right)\right]$ and $v(k)=[u(k) y(k)]^{\mathrm{T}}$,
} 
where $q_{z}=T \pi$ and $N_{z}=T H$. The existence of this property motivates the use of zonotopes to propagate the uncertainty in dynamic systems.

Using Theorem 1 , the zonotope $\mathbb{X}_{k+1}^{e}$ that bounds the trajectory of the system at instant $k+1$ is computed from the previous approximating zonotope at time instant $k, \mathbb{X}_{k}^{e}=\pi(k) \oplus H_{k} \beta^{m}$, using the natural interval extension of (42) as suggested by Moore [35] and the zonotope inclusion operator, as a generalization of Kuhn's method (see [37]):

$$
\mathbb{X}_{k+1}^{e}=\pi(k+1) \oplus H_{k+1} \beta^{r}
$$

where

$$
\begin{gathered}
\pi_{k+1}=\operatorname{mid}\left(A_{0}\left(\vartheta_{k}\right)\right) \pi(k)+\operatorname{mid}\left(B_{0}\left(\vartheta_{k}\right)\right) v(k) \\
H_{k+1}=\left[\begin{array}{lll}
J_{1} & J_{2} & J_{3}
\end{array}\right] \\
J_{1}=\operatorname{seg}\left(\diamond\left(A_{0}\left(\vartheta_{k}\right) H_{k}\right)\right) \\
J_{2}=\frac{\operatorname{diam}\left(A_{o}\left(\vartheta_{k}\right)\right)}{2} \pi(k) \\
J_{3}=\frac{\operatorname{diam}\left(B_{o}\left(\vartheta_{k}\right)\right)}{2} v(k)
\end{gathered}
$$

with $A_{0}\left(\vartheta_{k}\right) \triangleq \sum_{j=1}^{N} \alpha^{j}\left(p_{k}\right) A_{0, j}\left(\vartheta^{j}\right), B_{0}\left(\vartheta_{k}\right) \triangleq \sum_{j=1}^{N} \alpha^{j}\left(p_{k}\right) B_{0, j}\left(\vartheta^{j}\right), J_{1}$ is calculated using the zonotope inclusion operator and $\operatorname{seg}(\mathbb{X})=M$ considering that $\mathbb{X}=\pi \oplus M \beta^{m_{z}}$ is a zonotope (see Definition 4).

It is important to notice that the set of estimated states has an increasing number of segments generating the zonotope $\mathbb{X}_{k+1}^{e}$ using this method. In order to control the domain complexity, a reduction step is thus implemented. Here, we use the method proposed in [38] to reduce the zonotope complexity.

Analogously, Theorem 1 can be used to obtain the set $\mathbb{Y}_{k}^{e}$ from the set $\mathbb{X}_{k}^{e}$ through the expression for the estimated output (42).

\section{MINIMUM DETECTABLE FAULT}

\subsection{LPV fault residual sensitivity}

In general, the residual can be affected by different possible faults. To separate one fault from the others, the fault effect in the residual should be characterized. One way to characterize this effect is by using the fault residual sensitivity introduced by Gertler [2]

$$
S_{f}=\frac{\partial r}{\partial f}
$$

For a given fault $f$, the expression of residual sensitivity can be obtained using the residual internal form (33). It can be noticed that the sensitivity is a transfer function whose parameters change with the operating point parameterized by scheduling variable $p_{k}$ as in the LPV system (1) and (2) and that describes the effect on residual, $r$, of a given fault $f$.

Analyzing the residual internal form given by (33), and considering (46), the fault residual sensitivity for three cases can be obtained

- Output sensor fault sensitivity:

$$
S_{f_{y}}\left(q^{-1}, \tilde{\vartheta}_{k}\right)=\sum_{j=1}^{N} \alpha^{j}\left(p_{k}\right)\left[\left(I-H^{j}\left(q^{-1}, \vartheta^{j}\right)\right) G_{f y}^{j}\left(q^{-1}, \vartheta^{j}\right)\right]
$$


- Actuator fault sensitivity:

$$
S_{f_{a}}\left(q^{-1}, \tilde{\vartheta}_{k}\right)=\sum_{j=1}^{N} \alpha^{j}\left(p_{k}\right)\left[\left(I-H^{j}\left(q^{-1}, \vartheta^{j}\right)\right) G_{f a}^{j}\left(q^{-1}, \vartheta^{j}\right)\right]
$$

- Input sensor fault sensitivity:

$$
S_{f_{u}}\left(q^{-1}, \tilde{\vartheta}_{k}\right)=\sum_{j=1}^{N} \alpha^{j}\left(p_{k}\right)\left[-G_{f u}^{j}\left(q^{-1}, \vartheta^{j}\right)\right]
$$

Thus, the LPV residual internal form (33) can be written as follows:

$$
r(k)=r_{0}(k)+S_{f_{y}}\left(q^{-1}, \tilde{\vartheta}_{k}\right) f_{y}(k)+S_{f_{a}}\left(q^{-1}, \tilde{\vartheta}_{k}\right) f_{a}(k)+S_{f_{u}}\left(q^{-1}, \tilde{\vartheta}_{k}\right) f_{u}(k)
$$

where $S_{f y}, S_{f a}$ and $S_{f u}$ are the LPV residual sensitivity of an output sensor fault, actuator fault and input sensor fault, respectively.

\subsection{Minimum detectable fault}

According to Gertler [2], the minimum detectable fault ('triggering limit') corresponds to a fault that brings a residual to its threshold. The minimum detectable fault occurs when a fault brings the residual to its threshold. In other words

$$
r(k)=\bar{r}(k) \quad \text { or } \quad \underline{r}(k)
$$

where $\bar{r}(k)$ or $\underline{r}(k)$ can be given by (38) and (39). If the fault effects are included, the interval bound $\bar{r}(k)$ can be reformulated as:

$$
\begin{aligned}
\bar{r}(k)= & \max _{\underline{\vartheta}^{j} \leqslant \vartheta^{j} \leqslant \bar{\vartheta}^{j}, j=1, \ldots, N}\left\{\sum _ { j = 1 } ^ { N } \alpha ^ { j } ( p _ { k } ) \left[\Delta G^{j}\left(q^{-1}, \vartheta^{j}\right) u_{0}(k)+\Delta H^{j}\left(q^{-1}, \vartheta^{j}\right) y_{0}(k)\right.\right. \\
& +\Delta G^{j}\left(q^{-1}, \vartheta^{j}\right) F_{u}\left(\vartheta^{j}\right) f_{u}(k)+\Delta H^{j}\left(q^{-1}, \vartheta^{j}\right)\left(G_{f_{a}}\left(q^{-1}, \vartheta^{j}\right) f_{a}(k)\right. \\
& \left.\left.\left.+G_{f_{y}}\left(q^{-1}, \vartheta^{j}\right) f_{y}(k)\right)\right]\right\}
\end{aligned}
$$

Analogously, the threshold $\underline{r}(k)$ can be calculated according to (52) using the minimum function instead of maximum.

Substituting in the previous expression $r(k)$ by (33) and assuming that the maximum threshold $\bar{r}(k)$ is given by (52) leads to

$$
\begin{aligned}
& r_{0}(k)+\sum_{j=1}^{N} \alpha^{j}\left(p_{k}\right)\left[\left(I-H^{j}\left(q^{-1}, \vartheta^{j}\right)\right)\left(G_{f y}^{j}\left(q^{-1}, \vartheta^{j}\right) f_{y}(k)+G_{f a}^{j}\left(q^{-1}, \vartheta^{j}\right) f_{a}(k)\right)\right. \\
& \left.-G_{f u}^{j}\left(q^{-1}, \vartheta^{j}\right) f_{u}(k)\right] \\
& =\max _{\underline{\vartheta}^{j} \leqslant \vartheta^{j} \leqslant \bar{\vartheta}^{j}, j=1, \ldots, N}\left\{\sum_{j=1}^{N} \alpha^{j}\left(p_{k}\right)[\overbrace{\Delta G^{j}\left(q^{-1}, \vartheta^{j}\right) u_{0}(k)+\Delta H^{j}\left(q^{-1}, \vartheta^{j}\right) y_{0}(k)}^{\bar{r}_{0}(k)}\right. \\
& \left.\left.+\Delta G_{f_{u}}^{j}\left(q^{-1}, \vartheta^{j}\right) f_{u}(k)+\Delta H^{j}\left(q^{-1}, \vartheta^{j}\right)\left(G_{f_{a}}\left(q^{-1}, \vartheta^{j}\right) f_{a}(k)+G_{f_{y}}\left(q^{-1}, \vartheta^{j}\right) f_{y}(k)\right)\right]\right\}
\end{aligned}
$$


where $r_{0}(k)$ is a vector that contains the effect of the model parameter uncertainty on the residual. Therefore, to obtain the minimum detectable fault, the case $r_{0}(k)=0$ should be considered in the previous expression yielding to:

$$
\begin{aligned}
& \sum_{j=1}^{N} \alpha^{j}\left(p_{k}\right)\left[\left(I-H^{j}\left(q^{-1}, \vartheta_{0}^{j}\right)\right)\left(G_{f_{a}}\left(q^{-1}, \vartheta_{0}^{j}\right) f_{a}(k)+G_{f_{y}}\left(q^{-1}, \vartheta_{0}^{j}\right) f_{y}(k)\right)-G_{f u}^{j}\left(q^{-1}, \vartheta^{j}\right) f_{u}(k)\right] \\
& =\sum_{j=1}^{N} \alpha^{j}\left(p_{k}\right)\left[\bar{r}_{0}(k)+\Delta G_{f_{u}}^{j}\left(q^{-1}, \vartheta^{j}\right) f_{u}(k)+\Delta H^{j}\left(q^{-1}, \vartheta^{j}\right)\left(G_{f_{a}}\left(q^{-1}, \vartheta^{j}\right) f_{a}(k)\right.\right. \\
& \left.\left.\quad+G_{f_{y}}\left(q^{-1}, \vartheta^{j}\right) f_{y}(k)\right)\right]
\end{aligned}
$$

This expression can be particularized to evaluate the minimum detectable fault for output/input sensor or actuator faults. As example, the minimum output sensor fault case can be determined from

$$
\begin{aligned}
& \sum_{j=1}^{N} \alpha^{j}\left(p_{k}\right)\left[\left(I-H^{j}\left(q^{-1}, \vartheta_{0}^{j}\right)\right)\left(G_{f_{y}}\left(q^{-1}, \vartheta_{0}^{j}\right) f_{y}(k)\right)\right] \\
& \quad=\sum_{j=1}^{N} \alpha^{j}\left(p_{k}\right)\left[\bar{r}_{0}(k)+\Delta H^{j}\left(q^{-1}, \vartheta^{j}\right)\left(G_{f_{y}}\left(q^{-1}, \vartheta^{j}\right) f_{y}(k)\right)\right]
\end{aligned}
$$

that leads to

$$
\begin{aligned}
f_{y, p}^{\min }(k) & =\max _{\underline{\vartheta}^{j} \leqslant \vartheta^{j} \leqslant \bar{\vartheta}^{j}, j=1, \ldots, N}\left\{\sum_{j=1}^{N} \alpha^{j}\left(p_{k}\right)\left[\bar{r}_{0}(k)\left(\left(I-H^{j}\left(q^{-1}, \vartheta^{j}\right)\right) G_{f_{y}}\left(q^{-1}, \vartheta^{j}\right)\right)^{-1}\right]\right\} \\
& =\max _{\underline{\vartheta}^{j} \leqslant \vartheta^{j} \leqslant \bar{\vartheta}^{j}, j=1, \ldots, N}\left\{\sum_{j=1}^{N} \alpha^{j}\left(p_{k}\right)\left[\bar{r}_{0}(k)\left(S_{f_{y}}\left(q^{-1}, \vartheta^{j}\right)\right)^{-1}\right]\right\}
\end{aligned}
$$

assuming that $\left(S_{f_{y}}\left(q^{-1}, \vartheta^{j}\right)\right)^{-1}$ exists and is stable. ${ }^{\S}$

Notice that subindex ' $p$ ' in the previous expression indicated that the minimum detectable fault has been computed considering the upper bound of the residual $\bar{r}(k)$. Analogously, a negative value could have been computed considering the lower bound $\underline{r}(k)$.

Analogously, applying the analysis procedure used in the output sensor fault, the minimum detectable for actuator fault and input sensor fault can be estimated [29].

\section{CASE 1: QUADRUPLE-TANK SYSTEM}

\subsection{Description of quadruple-tank system}

As an illustrative example, the four-interconnected tank system proposed in [23] is used to validate the algorithms and results obtained along the paper. This system is proposed as multivariable control benchmark where the level in the lower tanks is controlled with the two pumps. The process is described by the non-linear model (B1)-(B4) presented in Appendix B, where the inputs are $v_{1}$ and $v_{2}$ (input voltages to the pumps) and the outputs are $h_{1}, h_{2}, h_{3}$ and $h_{4}$ (level measurement devices). A schematic diagram of the process is shown in Figure 3.

\subsection{LPV model and interval LPV observer design}

The procedure to obtain an interval polytopic LPV model for the quadruple-tank system is described.

\footnotetext{
$\overline{\S \S\left(S_{f}\left(q^{-1}, \vartheta^{j}\right)\right)^{-1}}$ is computed elementwise. When some element of $\left(S_{f}\left(q^{-1}, \vartheta^{j}\right)\right)^{-1}$ is non-causal, Peng et al. [39]
} proposed multiplying by it the required number of delays, such that the causality property is satisfied. 




Figure 3. Quadruple-tank process.

LPV model representation: Following the approach introduced by Shamma and Cloutier [6], named state transformation, a discrete-time LPV model can be obtained for the quadruple-tank system (B1)-(B4) (see Appendix B) using a sampling time equal to $T_{\mathrm{s}}=0.5 \mathrm{~s}$ as follows:

$$
\left[\begin{array}{c}
h_{1}(k+1)-h_{1}^{\mathrm{eq}}\left(p_{k+1}\right) \\
h_{2}(k+1)-h_{2}^{\mathrm{eq}}\left(p_{k+1}\right)
\end{array}\right]=A\left(\tilde{\vartheta}_{k}\right)\left[\begin{array}{l}
h_{1}(k)-h_{1}^{\mathrm{eq}}\left(p_{k}\right) \\
h_{2}(k)-h_{2}^{\mathrm{eq}}\left(p_{k}\right)
\end{array}\right]+B\left(\tilde{\vartheta}_{k}\right)\left[\begin{array}{c}
v_{1}(k)-v_{1}^{\mathrm{eq}}\left(p_{k}\right) \\
v_{2}(k)-v_{2}^{\mathrm{eq}}\left(p_{k}\right)
\end{array}\right]
$$

where

$$
A\left(\tilde{\vartheta}_{k}\right)=\left[\begin{array}{cc}
\tilde{\vartheta}_{1}(k) & 0 \\
0 & \tilde{\vartheta}_{2}(k)
\end{array}\right], \quad B\left(\tilde{\vartheta}_{k}\right)=\left[\begin{array}{cc}
\tilde{\vartheta}_{3}(k) & \tilde{\vartheta}_{4}(k) \\
\tilde{\vartheta}_{5}(k) & \tilde{\vartheta}_{6}(k)
\end{array}\right]
$$

and $\tilde{\vartheta}_{k}=\left[\tilde{\vartheta}_{1}(k), \tilde{\vartheta}_{2}(k), \tilde{\vartheta}_{3}(k), \tilde{\vartheta}_{4}(k), \tilde{\vartheta}_{5}(k), \quad \tilde{\vartheta}_{6}(k)\right]^{\mathrm{T}}$ (the detailed expressions can be found in Appendix B).

Notice that this approach is based on rewriting the plant in a form where non-linear terms are hidden with newly defined time-varying parameters $\vartheta_{k}$ without involving any plant linearization $[40,41]$. The time-varying parameters are parameterized with respect to scheduling variables $p_{k}$ that correspond to measured outputs (in this case $h_{3}(k)$ and $h_{4}(k)$ ).

Identification of LPV model: The scheduling functions $f\left(p_{k}\right)$ for the LPV parameters are approximated by polynomials whose coefficients are estimated following the procedure described in [27] using data taken from the simulation of the non-linear model at different operating points obtained by griding the range of the scheduling variables: $h_{3} \in[1,20]$ and $h_{4} \in[1,20]$. After the identification process, the modeling error for each parameter is bounded as follows:

$$
\bar{\varepsilon}_{i}=\max _{p_{k} \in[\underline{p}, \bar{p}]}\left|\tilde{\vartheta}_{i}(k)-f_{i}\left(p_{k}\right)\right|, \quad i=1, \ldots, 6
$$

and $\bar{\varepsilon}_{i}=-\underline{\varepsilon}_{i}$. This modeling error for each parameter is $\varepsilon_{1}(k) \in[-0.0064,0.0064], \varepsilon_{2}(k) \in$ $[-0.0044,0.0044], \varepsilon_{3}(k) \in[-0.0132,0.0132], \varepsilon_{4}(k) \in[-0.0189,0.0189], \varepsilon_{5}(k) \in[-0.014,0.014]$, $\varepsilon_{6}(k) \in[-0.02,0.02]$.

Reduced LPV model: The LPV parameter vector is $\vartheta(k)=\left[\begin{array}{llllll}\vartheta_{1}(k) & \vartheta_{2}(k) & \vartheta_{3}(k) & \vartheta_{4}(k) & \vartheta_{5}(k) & \vartheta_{6}(k)\end{array}\right]$ (i.e. $l=6$ varying parameters) corresponding to the model (58), which depends on $h_{3}(k)$ and $h_{4}(k)$. Now, the parameter set mapping approach proposed by Kwiatkowski and Werner [42] (see Appendix A) is applied to determine a reduced number of LPV parameters using the principal component analysis. As a result, the number of LPV parameters is reduced to $m=3$ parameters $\left(\phi(k)=\left[\begin{array}{lll}\phi_{1}(k) & \phi_{2}(k) & \phi_{3}(k)\end{array}\right]^{\mathrm{T}}\right)$. The quality of this approximation evaluated using (A8) gives $v_{m}=$ 99\% of explained variance. 
Vertices of polytopic LPV model: The simplest polytopic approximation, known as bounding box approach, relies on bounding each LPV parameter by an interval [5]. An alternative approach known as small convex hull proposed by Kumar and Anderson [43] relies on using a polytope instead of an interval box.

Here, the small convex hull algorithm proposed by Biannic [44] is used. This algorithm generates a polytopic convex hull from the bounding box approach by the division of the original bounding box in smaller bounding boxes. Then, the set of smaller bounding boxes that do not contain parameters is removed. Finally, the set of the small bounding boxes containing parameters are used to obtain the small convex hull through the quick hull algorithrn [45]. Using this procedure it is guaranteed that true parameters will always be inside the computed domain. Moreover, this domain will be inflated to consider the parameter uncertainty (3) introduced by the experimental calibration of the scheduling function $f\left(p_{k}\right)$.

Figure 4(a) presents a comparison between the bounding box [5] and small hull approach [43]. This figure also shows the values of $\vartheta_{1}$ and $\vartheta_{2}$ for a representative set of operating points obtained from a sparse gridding of the operating range. This gives an idea of the quality of the approximation. Each operating point is defined by the scheduling variables $\left(h_{3}, h_{4}\right)$ and gives a value for the parameter vector $\left(\vartheta_{1}, \vartheta_{2}\right)$, where $\vartheta_{1}=f_{1}\left(h_{3}, h_{4}\right)$ and $\vartheta_{2}=f_{2}\left(h_{3}, h_{4}\right)$. It can be noticed that both techniques provide a convex hull that contains all operating points, but the small hull approach produces a sharper approximation compared with the bounding box approach.

The error between the LPV model of the quadruple-tank system and polytopic LPV model is evaluated with the following expression:

$$
\eta_{\vartheta, i}=\max _{p_{k} \in[\underline{p}, \bar{p}]}\left|\tilde{\vartheta}_{i}(k)-\sum_{j=1}^{N} \alpha^{j}\left(p_{k}\right) \vartheta_{i}^{j}\right|, \quad i=1, \ldots, 6
$$

The LPV model without reducing the number of LPV parameters (i.e. $l=6$ parameters) is presented in the first column of the bounding box case in Table I. In this case the number of vertices is $N=2^{l}=64$. The second column of the bounding box case presents the results for the LPV model with reduced number of parameters (i.e. $m=3$ parameters, $N=2^{m}=8$ ). The small convex hull approach is applied to the reduced parameter LPV model using different number of bounding boxes to approximate the exact LPV parameter domain following the algorithm of [44]. It can be seen that the maximum parameter error $\left(\max \eta_{\vartheta, i}\right)$ decreases by increasing number of vertices. Notice that comparing the error using the bounding box case with $N=64$ and the small convex hull case with $N=98$, there is no significant difference in the number of vertices. But, the error presented in the small convex hull case is smaller than the error presented in the bounding box. Figure 4(b) presents the small convex hull used to implement the polytopic LPV model with $N=98$. It is also worth to point that the number of vertices tends to not increase when augmenting the number of bounding boxes used in the small hull algorithm. This allows to determine the number of models to be used in the polytopic model.
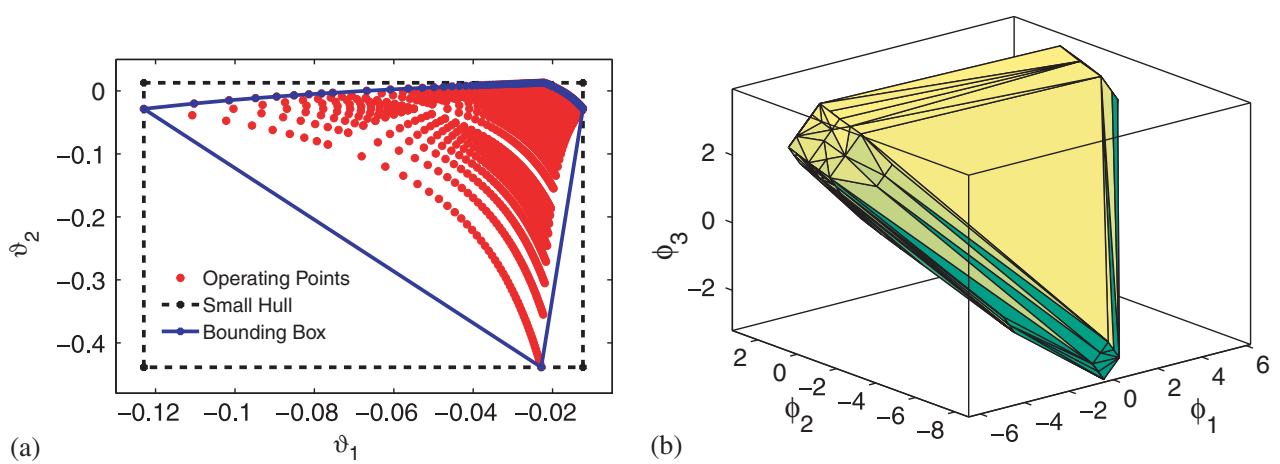

Figure 4. (a) LPV modeling technique in two dimensions for the bounding box and small hull approach and (b) small convex hull in three dimensions $\left(\phi_{1}, \phi_{2}, \phi_{3}\right)$ with 98 vertices. 
S. MONTES DE OCA, V. PUIG AND J. BLESA

Table I. Different number of $N$ models.

\begin{tabular}{|c|c|c|c|c|c|c|c|}
\hline \multirow[b]{2}{*}{$\begin{array}{l}\text { Number of bounding boxes } \\
\text { Vertices }\end{array}$} & \multicolumn{2}{|c|}{ Bounding box } & \multicolumn{5}{|c|}{ Small convex hull } \\
\hline & $\overline{64}$ & $\overline{8}$ & $\begin{array}{c}729 \\
68\end{array}$ & $\begin{array}{c}4096 \\
83\end{array}$ & $\begin{array}{c}15625 \\
92\end{array}$ & $\begin{array}{c}46656 \\
97\end{array}$ & $\begin{array}{c}1000000 \\
98\end{array}$ \\
\hline$\eta_{9,1}$ & 0.0424 & 0.0323 & 0.0424 & 0.0424 & 0.0424 & 0.0139 & 0.0129 \\
\hline$\eta_{\vartheta, 2}$ & 0.0289 & 0.0380 & 0.0289 & 0.0289 & 0.0289 & 0.0096 & 0.0086 \\
\hline$\eta_{\vartheta, 3}$ & 0.1948 & 0.1243 & 0.1121 & 0.0744 & 0.0546 & 0.0385 & 0.0382 \\
\hline$\eta_{\vartheta, 4}$ & 0.2784 & 0.0987 & 0.0928 & 0.0696 & 0.0557 & 0.0555 & 0.0319 \\
\hline$\eta_{\vartheta, 5}$ & 0.2064 & 0.1051 & 0.1188 & 0.0788 & 0.0579 & 0.0393 & 0.0419 \\
\hline$\eta_{\vartheta, 6}$ & 0.2949 & 0.2267 & 0.0983 & 0.0737 & 0.0590 & 0.0562 & 0.0268 \\
\hline $\max \eta_{\vartheta, i}$ & 0.2949 & 0.2267 & 0.1188 & 0.0788 & 0.0590 & 0.0562 & 0.0419 \\
\hline
\end{tabular}

Table II. Description of the fault scenarios.

\begin{tabular}{lc}
\hline Fault & Description \\
\hline$f_{a, \ell}$ & Additive fault in the pump $\ell$ \\
$f_{h, \ell}$ & Additive fault in the level measurement device of tank $\ell$ \\
\hline
\end{tabular}

Table III. Minimum detectable fault for different operating points.

\begin{tabular}{|c|c|c|c|c|c|c|c|c|c|c|c|c|}
\hline & \multicolumn{6}{|c|}{$\mathscr{O} \mathscr{P}_{1}\left(h_{3}=0.31 \mathrm{~V}\right.$ and $\left.h_{4}=0.31 \mathrm{~V}\right)$} & \multicolumn{6}{|c|}{$\mathscr{O} \mathscr{P}_{2}\left(h_{3}=1.25 \mathrm{~V}\right.$ and $\left.h_{4}=1.25 \mathrm{~V}\right)$} \\
\hline & $f_{h_{1}}$ & $f_{h_{2}}$ & $f_{h_{3}}$ & $f_{h_{4}}$ & $f_{a_{1}}$ & $f_{a_{2}}$ & $f_{h_{1}}$ & $f_{h_{2}}$ & $f_{h_{3}}$ & $f_{h_{4}}$ & $f_{a_{1}}$ & $f_{a_{2}}$ \\
\hline$r_{1}$ & -0.25 & 0 & -0.05 & -0.08 & -0.79 & -0.24 & -0.56 & 0 & -0.13 & -0.20 & -0.93 & -0.28 \\
\hline$r_{1}$ & 0.230 & 0 & 0.060 & 0.096 & 0.860 & 0.265 & 0.540 & 0.000 & 0.138 & 0.218 & 0.979 & 0.301 \\
\hline$r_{2}$ & 0 & -0.33 & -0.06 & -0.07 & -0.16 & -0.21 & 0 & -0.75 & -0.15 & -0.16 & -0.18 & -0.24 \\
\hline$r_{2}$ & 0 & 0.307 & 0.071 & 0.076 & 0.174 & 0.229 & 0 & 0.721 & 0.163 & 0.174 & 0.198 & 0.261 \\
\hline$r_{3}$ & 0 & 0 & -0.03 & 0 & 0 & -0.08 & 0 & 0 & -0.08 & 0 & 0 & -0.09 \\
\hline$r_{3}$ & 0 & 0 & 0.032 & 0 & 0 & 0.075 & 0 & 0 & 0.078 & 0 & 0 & 0.089 \\
\hline$r_{4}$ & 0 & 0 & 0 & -0.03 & -0.0 & 0 & 0 & 0 & 0 & -0.08 & -0.05 & 0 \\
\hline$r_{4}$ & 0 & 0 & 0 & 0.032 & 0.036 & 0 & 0 & 0 & 0 & 0.078 & 0.044 & 0 \\
\hline
\end{tabular}

Implementation of interval LPV observer: The polytopic interval LPV observer (10) and (11) is implemented using $N=98$ models provided by the small hull approach and taking into account modeling uncertainty $(60)$.

The observer design procedure proposed in Section 2.3 was applied to obtain the gain $L\left(\vartheta_{k}\right)$, such that the poles are in the LMI disk region $\mathscr{D}$ with the parameters $q=-0.5$ and $r=0.25$. This procedure results in $N=98$ observer gains $L_{j}\left(\vartheta^{j}\right)$ that they will be interpolated as $L\left(\vartheta_{k}\right)=$ $\sum_{j=1}^{N} \alpha^{j}\left(p_{k}\right) L_{j}\left(\vartheta^{j}\right)$.

\subsection{Fault scenarios}

The mathematical model (B1)-(B4) of quadruple-tank process has been modified in order to include a set of typical faults. The faults are described in Table II.

In the following, only fault detection results using the interval LPV observer in two of these fault scenarios are presented. For illustrative purposes, in the first scenario, the fault detection test is based on generating the output prediction intervals according to (28) as presented in Section 3.1. The second scenario implements the fault detection test based on residual adaptive thresholds (36) presented in Section 3.2.

The minimum detectable faults using the interval LPV observer are determined using the expressions obtained in Section 5.2 based on the fault sensitivity analysis. Table III presents the minimum detectable fault corresponding to two different operating points $(\mathscr{O P})$. This table shows the effect 

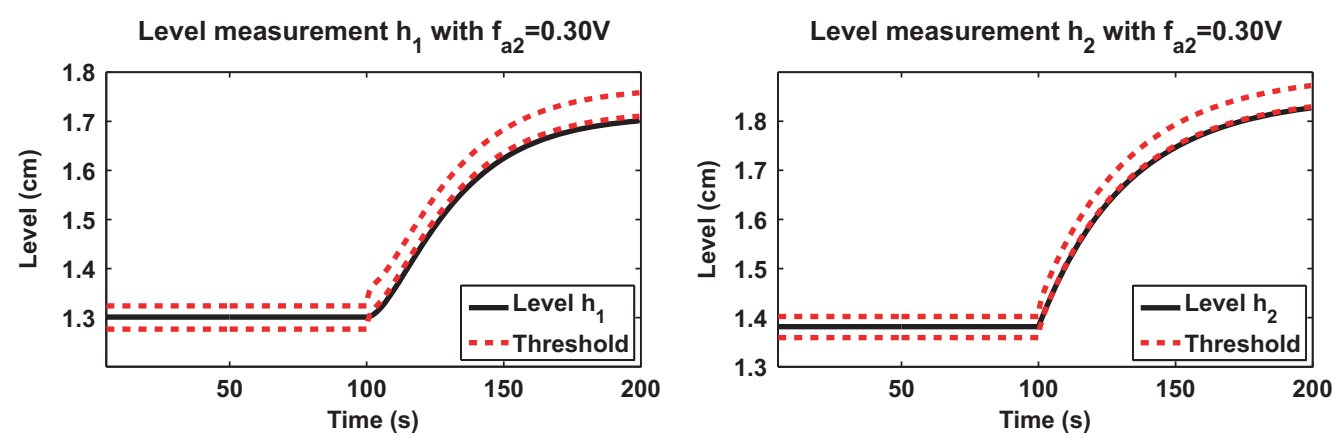

Figure 5. Level measurement of $h_{1}, h_{2}$ and the output prediction interval (fault scenario with $f_{v_{2}}=0.3 \mathrm{~V}$ ).
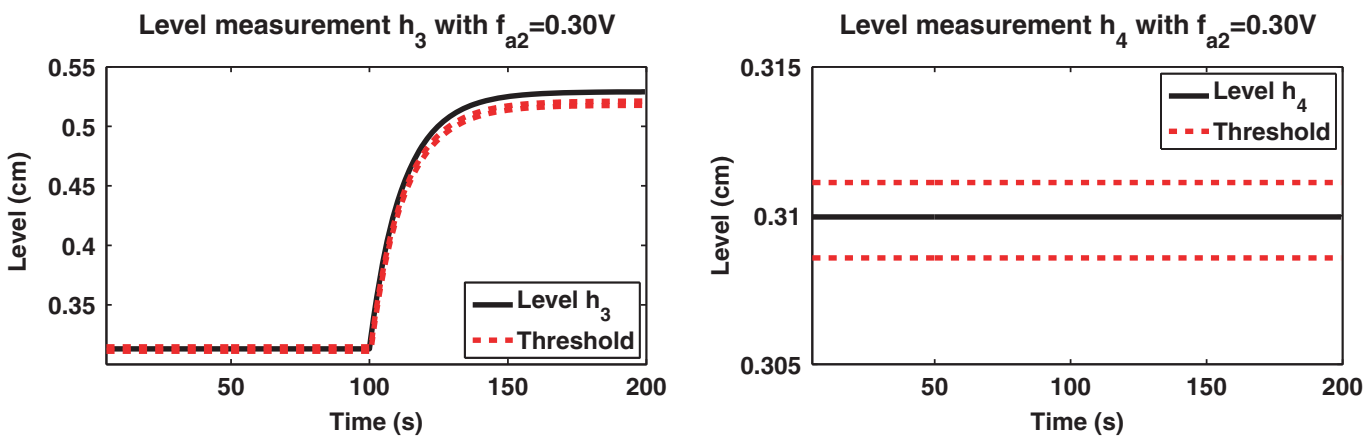

Figure 6. Level measurement of $h_{3}, h_{4}$ and the output prediction interval (fault scenario with $f_{v_{2}}=0.3 \mathrm{~V}$ ).

of the operating point in the minimum detectable fault. It can be seen that the minimum detectable fault is different at each operating point. This justifies the use of an interval LPV observer.

6.3.1. Fault scenario 1. Quadruple-tank process with an actuator fault $\left(f_{a_{2}}=0.3 \mathrm{~V}\right)$.

A fault in Pump 2 (actuator fault: $f_{a_{2}}=0.3 \mathrm{~V}$ ) at time $t=100 \mathrm{~s}$ is introduced. This fault appears when the system is in the first operating point $\left(\mathcal{O} \mathscr{P}_{1}\right)$. The column $f_{a_{2}}$ in Table III shows that the minimum detectable fault for this operating point is $f_{a_{2}, r_{1}}^{\min }=0.265 \mathrm{using}$ the residual $r_{1}, f_{a_{2}, r_{2}}^{\min }=$ 0.229 using the residual $r_{2}$ and $f_{a_{2}, r_{3}}^{\min }=0.075$ using the residual $r_{3}$. The residual $r_{4}$ is not affected by this fault. Since the size of the fault is $0.3 \mathrm{~V}$, all the residuals sensitive to the fault will detect it. This can be observed in Figure 5 where the output prediction interval is presented for each measurement $\left(h_{1}, h_{2}, h_{3}\right.$ and $\left.h_{4}\right)$. In particular, Figures 5 and 6 show that the measurements $h_{1}, h_{2}$ and $h_{3}$ are outside the output prediction intervals what allow to detect the fault. Output prediction intervals are calculated using the interval LPV observer (24).

6.3.2. Fault scenario 2. Quadruple-tank process with an output sensor fault $\left(f_{h_{4}}=0.2 \mathrm{~cm}\right)$.

A fault in the level measurement device of tank $4 h_{4}\left(f_{h_{4}}=0.2 \mathrm{~cm}\right)$ at time $t=100 \mathrm{~s}$ is introduced. As in the previous scenario, the system is in the first operating point $\left(\mathcal{O} \mathscr{P}_{1}\right)$. According to Table III, the minimum detectable fault is $f_{h_{4}, r_{1}}^{\min }=0.096 \mathrm{using}$ the residual $r_{1}, f_{h_{4}, r_{2}}^{\min }=0.076 \mathrm{using}$ the residual $r_{2}$ and $f_{h_{4}, r_{4}}^{\min }=0.032$ using the residual $r_{4}$. The residual $r_{3}$ is not affected by this fault. Figures 7 and 8 show the residual evaluation and their prediction bounds (adaptive threshold). It can be seen that residuals corresponding to measurements $h_{1}, h_{2}$ and $h_{4}$ detect the fault presence. The residual (32) is implemented using the interval LPV observer (24) and evaluated using the adaptive threshold (36). 

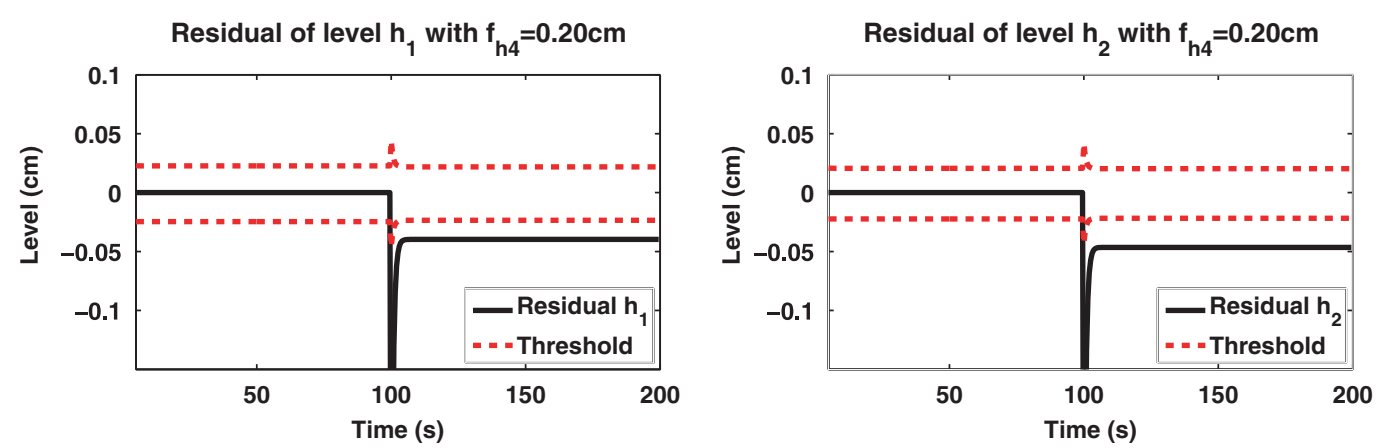

Figure 7. Residual signal of $h_{1}, h_{2}$ and the adaptive threshold (fault scenario with $f_{h_{4}}=0.2 \mathrm{~cm}$ ).
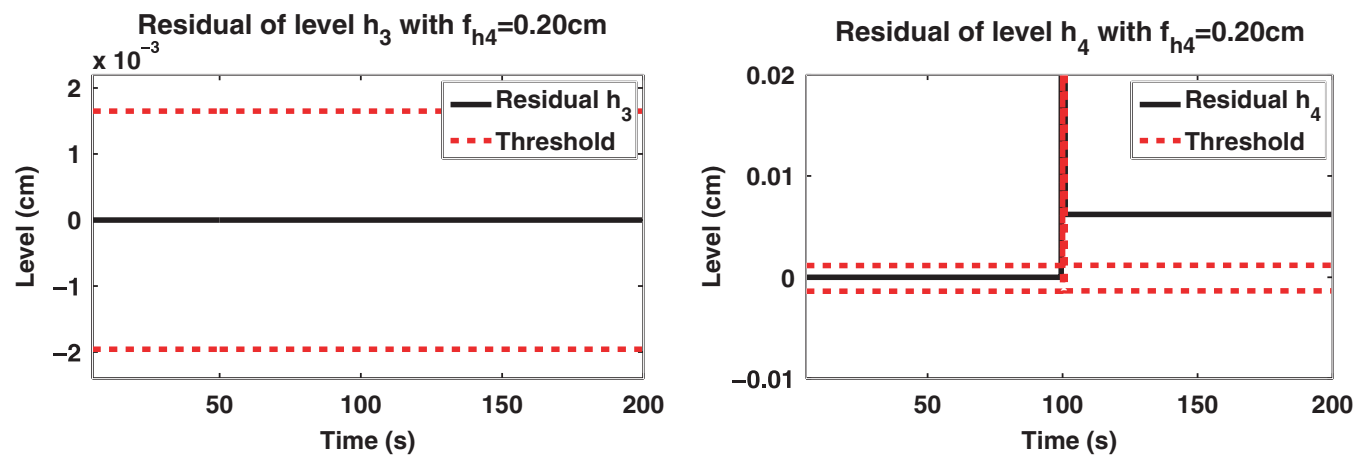

Figure 8. Residual signal of $h_{3}, h_{4}$ and the adaptive threshold (fault scenario with $f_{h_{4}}=0.2 \mathrm{~cm}$ ).

\section{CASE 2: TWIN-ROTOR MIMO SYSTEM}

A second example based on the twin-rotor MIMO system to validate the proposed LPV interval observer approach is used. This case is used to prove its validity when applied to a higher order system with less measurements than states.

\subsection{Description of twin-rotor MIMO system}

The TRMS is a laboratory setup developed by Feedback Instruments Limited for advanced control experiments. The system is perceived as a challenging control engineering problem due to its high non-linearity, cross-coupling between its two axes, and inaccessibility of some of its states through measurements. The TRMS mechanical unit has two rotors (the main and tail rotors) driven by DC motors placed on a beam together with a counterbalance whose arm with a weight at its end is fixed to the beam at the pivot (Figure 9). The TRMS can rotate freely both in the horizontal and vertical planes.

The system input vector is $u=\left[u_{\mathrm{t}}, u_{\mathrm{m}}\right]^{\mathrm{T}}$, where $u_{\mathrm{t}}$ is the input voltage of the tail motor and $u_{\mathrm{m}}$ is the input voltage of the main motor. The system states are $x=\left[i_{a h}, \omega_{h}, \Omega_{h}, \theta_{h}, i_{a v}, \omega_{v}, \Omega_{v}, \theta_{v}\right]^{\mathrm{T}}$, where $i_{a h / v}$ is the armature current of tail/main rotor, $\omega_{t / m}$ is the rotational velocity of the tail/main rotor, $\Omega_{h / v}$ is the angular velocity around the horizontal/vertical axis and $\theta_{h / v}$ is the azimuth/pitch angle of beam. Finally, the system measured outputs are $y=\left[\theta_{h}, \theta_{v}\right]^{\mathrm{T}}$.

\subsection{The TRMS LPV model}

The mathematical model of TRMS is given by a set of non-linear differential equations (C1)-(C8), which is presented in Appendix C. Following [46], the LPV representation of this model can be 


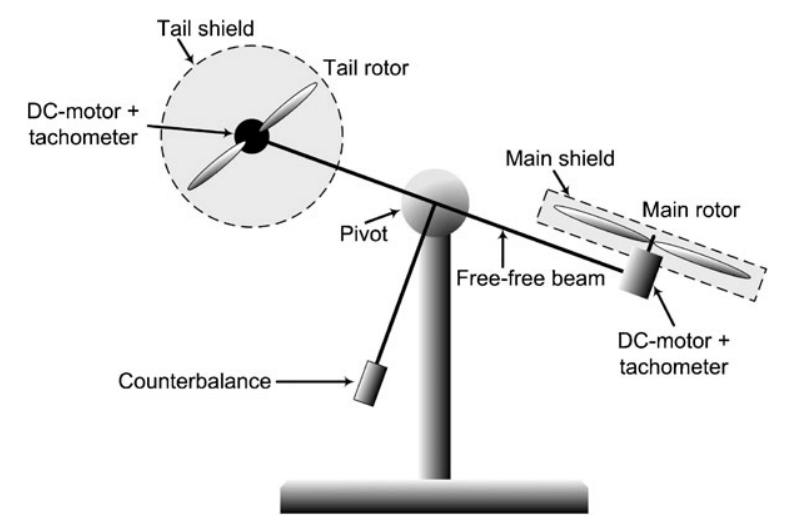

Figure 9. Components of the twin-rotor MIMO system.

obtained from the non-linear model (C1)-(C8) that can be expressed as follows:

$$
\begin{aligned}
x(k+1) & =A\left(\tilde{\vartheta}_{k}\right) x(k)+B u(k) \\
y(k) & =C x(k)
\end{aligned}
$$

where

$$
\begin{aligned}
& A\left(\vartheta_{k}\right)=\left[\begin{array}{cccccccc}
a_{11} & a_{12} & 0 & 0 & 0 & 0 & 0 & 0 \\
a_{21} & \tilde{\vartheta}_{1}(k) & 0 & 0 & 0 & 0 & 0 & 0 \\
0 & \tilde{\vartheta}_{2}(k) & \tilde{\vartheta}_{3}(k) & \tilde{\vartheta}_{4}(k) & \tilde{\vartheta}_{5}(k) & \tilde{\vartheta}_{6}(k) & \tilde{\vartheta}_{7}(k) & 0 \\
0 & 0 & a_{43} & 0 & 0 & 0 & 0 & 0 \\
0 & 0 & 0 & 0 & a_{55} & a_{56} & 0 & 0 \\
0 & 0 & 0 & 0 & a_{65} & \tilde{\vartheta}_{8}(k) & 0 & 0 \\
a_{71} & \tilde{\vartheta}_{9}(k) & \tilde{\vartheta}_{10}(k) & 0 & 0 & \tilde{\vartheta}_{11}(k) & a_{77} & \tilde{\vartheta}_{12}(k) \\
0 & 0 & 0 & 0 & 0 & 0 & a_{87} & 0
\end{array}\right] \\
& B=\left[\begin{array}{cccccccc}
b_{11} & 0 & 0 & 0 & 0 & 0 & 0 & 0 \\
0 & 0 & 0 & 0 & b_{52} & 0 & 0 & 0
\end{array}\right]^{\mathrm{T}}, \quad C=\left[\begin{array}{cccccccc}
0 & 0 & 0 & 1 & 0 & 0 & 0 & 0 \\
0 & 0 & 0 & 0 & 0 & 0 & 0 & 1
\end{array}\right]^{\mathrm{T}}
\end{aligned}
$$

with $\tilde{\vartheta}(k)=\left[\tilde{\vartheta}_{1}(k), \ldots, \tilde{\vartheta}_{12}(k)\right]^{\mathrm{T}}$ being the LPV parameter vector that uses as scheduling variables the available measured outputs (i.e. the azimuth angle $\theta_{h}(k)$ and the pitch angle of the beam $\theta_{v}(k)$ ).

To obtain the polytopic LPV model (4) and (5) the same procedure proposed in the quadrupletank example presented in Section 6 is used. As a result, the LPV parameter vector $\vartheta(k)=$ $\left[\vartheta_{1}(k), \ldots, \vartheta_{12}(k)\right]$ is reduced to $\phi(k)=\left[\phi_{1}(k), \ldots, \phi_{4}(k)\right]$. In this case the small convex hull is applied and the number of vertices is $N=847$. Finally, the interval LPV observer is designed, such that the poles are in a disk region of $\mathscr{D}$ with $q=-0.95, r=0.04$.

\subsection{Fault scenarios}

Two selected fault scenarios are used to illustrate the performance of the proposed approach.

7.3.1. Fault scenario 1. Fault in the pitch angle of the beam $\left(f_{\vartheta_{v}}=0.05\right)$.

A fault in the pitch angle of the beam $\left(f_{\vartheta_{v}}=0.05\right)$ at time $t=20 \mathrm{~s}$ is introduced. The output prediction interval (28) is generated using the interval LPV observer (24). Figure 10 shows that the measurements are outside its prediction interval what allows to detect the output sensor fault at time $t=24 \mathrm{~s}$ in the case of $\vartheta_{h}$ and at time $t=20 \mathrm{~s}$ in the case of $\vartheta_{v}$. 

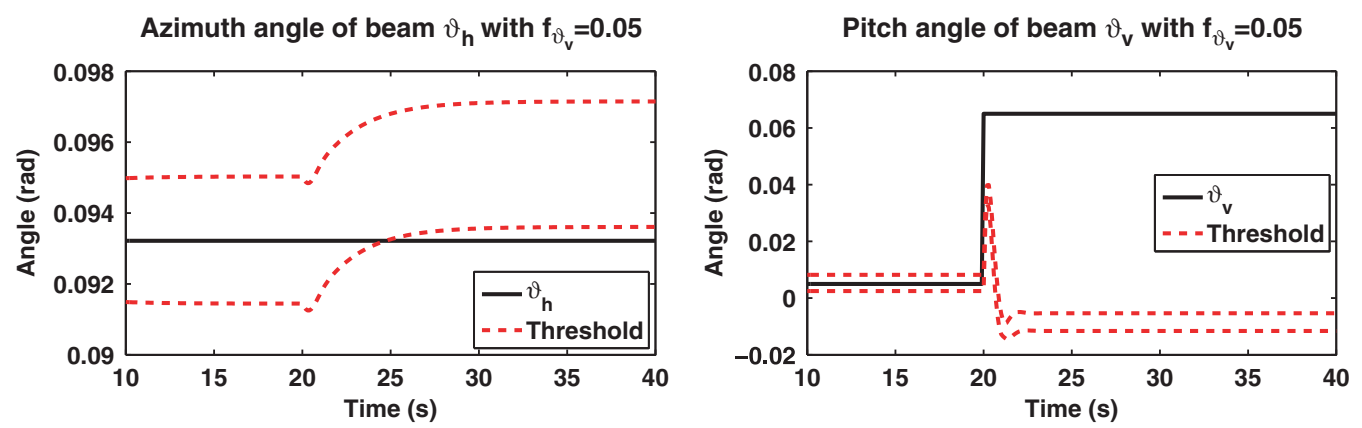

Figure 10. The azimuth angle of the beam $\vartheta_{h}$ and pitch angle of the beam $\vartheta_{v}$ and its the output prediction interval (fault scenario with $f_{\vartheta_{v}}=0.05$ ).
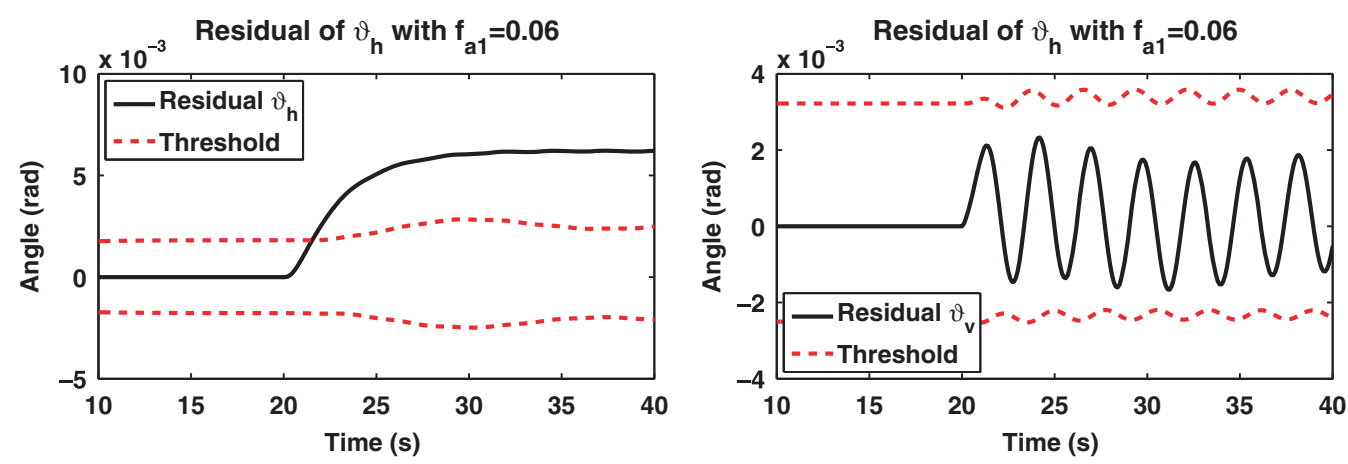

Figure 11. Residual of azimuth/pitch angle of the beam and the adaptive threshold (fault scenario with $f_{a_{1}}=0.06$ ).

\subsubsection{Fault scenario 2. Fault in the tail rotor $\left(f_{a_{1}}=0.06\right)$.}

A fault in the tail rotor $\left(f_{a_{1}}=0.06\right)$ at time $t=20 \mathrm{~s}$ is introduced. This scenario illustrates the residual evaluation (32) using the adaptive threshold (36). Figure 11 shows the residual evaluation and their threshold. It can be seen that the residual corresponding to measurement $\vartheta_{h}$ detects the fault presence at time $t=21.5 \mathrm{~s}$.

\section{CONCLUSIONS}

In this paper, robust fault detection based on adaptive threshold generation for non-linear systems that can be described by means of an LPV model has been addressed. The interval LPV model is obtained from a non-linear system taking into account the model uncertainty in the LPV parameter identification. The parameter uncertainty is bounded using intervals.

The non-linear system can be monitored using an interval LPV observer with the Luenberger structure considering parameter uncertainty what allows to generate an adaptive threshold that it is used to implement the robust fault detection test.

The interval LPV observer could work with either an interval LPV model or its polytopic approximation. However, the design of the LPV observer gain using the LMI pole placement requires that LPV model should be approximated by a polytopic LPV model. For this reason, the observer is designed using the polytopic LPV model.

Fault detection limitations of the proposed approach have been characterized by means of minimum detectable faults that are determined using fault sensitivity analysis.

Two examples, one based on the four tanks system and another based on a two-degree of freedom helicopter, have been used to demonstrate the effectiveness and performance of the proposed approach. 
As further work, the noise effect will be considered. The noise effect should be considered in the observer design and in the fault detection test. Although considering the noise effect in the detection test is straightforward if the noise is assumed bounded (as parameter uncertainty). However, the design of the LPV observer considering the noise and parameter uncertainty at the same time is a theoretical open issue [30]. The proposed method will be extended to address fault isolation tasks making use of the sensitivity analysis presented in this paper. Preliminary results are presented in [47].

\section{APPENDIX A: PARAMETER SET MAPPING}

Parameter set mapping (PSM) was introduced in [42] as a procedure for constructing a coordinate transformation for a given LPV model that leads to a tighter parameter set or lower dimensional approximations of this set. The procedure requires to obtain a set of operating points of the scheduling variables that can be used to generate the following $l \times M$ data matrix:

$$
\Xi=\left[\vartheta(1) \ldots \vartheta\left(M T_{\mathrm{s}}\right)\right]
$$

The rows $\Xi_{i}$ of the data matrix need to be normalized

$$
\Xi_{i}^{n}=\mathscr{N}_{i}\left(\Xi_{i}\right), \quad \Xi_{i}=\mathscr{N}_{i}^{-1}\left(\Xi_{i}^{n}\right)
$$

resulting in a normalized data matrix $\Xi^{n}=\mathscr{N}(\Xi)$. Now, the PCA is applied to the normalized data. Introducing the singular value decomposition

$$
\Xi^{n}=\left[\begin{array}{ll}
U_{s} & U_{n}
\end{array}\right]\left[\begin{array}{ccc}
\Sigma_{s} & 0 & 0 \\
0 & \Sigma_{n} & 0
\end{array}\right]\left[\begin{array}{c}
V_{s}^{\mathrm{T}} \\
V_{n}^{\mathrm{T}}
\end{array}\right]
$$

if the normalized data are correlated, some singular values are small compared with the others, where $U_{s}, \Sigma_{s}$ and $V_{s}$ denote the $m$ significant parameters singular values. Assuming that the less significant singular values can be neglected, (A3) is approximated as follows:

$$
\hat{\Xi}^{n}=U_{s} \Sigma_{s} V_{s}^{\mathrm{T}} \approx \Xi^{n}
$$

The comparison of $\hat{\Xi}^{n}$ and $\Xi^{n}$ allows to assess the quality of the approximation, and (by choosing the $m$ number of parameters) to trade accuracy of the model against complexity. The matrix $U_{s} \in \mathfrak{R}^{l \times m}$ represents a basis of the significant column space of the data matrix $\Xi^{n}$, and can be used to obtain a reduced mapping $\vartheta_{k}$ to $\phi_{k}$ by computing:

$$
\phi_{k}=U_{s}^{\mathrm{T}} \mathscr{N}\left(\vartheta_{k}\right)
$$

Considering the LPV system (4) and (5), the model derived from the representation map is given by

$$
\left(\begin{array}{ll}
A\left(\tilde{\vartheta}_{k}\right) & B\left(\tilde{\vartheta}_{k}\right) \\
C\left(\tilde{\vartheta}_{k}\right) & D\left(\tilde{\vartheta_{k}}\right)
\end{array}\right) \approx\left(\begin{array}{ll}
\hat{A}\left(\phi_{k}\right) & \hat{B}\left(\phi_{k}\right) \\
\hat{C}\left(\phi_{k}\right) & \hat{D}\left(\phi_{k}\right)
\end{array}\right)=\left(\begin{array}{ll}
A\left(\hat{\vartheta}_{k}\right) & B\left(\hat{\vartheta}_{k}\right) \\
C\left(\hat{\vartheta}_{k}\right) & D\left(\hat{\vartheta}_{k}\right)
\end{array}\right)
$$

where

$$
\hat{\vartheta}_{k}=\mathscr{N}^{-1}\left(U_{s} \phi_{k}\right)
$$

and $\mathscr{N}^{-1}$ denotes row-wise rescaling. At any given time, (A5) can be used to compute the reduced parameter vector, whereas (A6) together with (A7) can be used to generate the approximate LPV model.

As a measure of the quality of the approximation, the fraction of total variation $v_{m}$ that can be determined by the singular values $\sigma_{i}$ in (A3) is considered:

$$
v_{m}=\frac{\sum_{i=1}^{m} \sigma_{i}^{2}}{\sum_{i=1}^{l} \sigma_{i}^{2}}
$$




\section{APPENDIX B: LPV MODEL OF QUADRUPLE-TANK SYSTEM}

The target of the quadruple-tank system [23] is to control the level in the lower two tanks with two pumps. The process inputs are $v_{1}$ and $v_{2}$ (input voltages to the pumps) and the outputs are $h_{1}, h_{2}, h_{3}$ and $h_{4}$ (voltages from level measurement devices). A schematic diagram of the process is shown in Figure 3. The mass balances and Bernoulli's law yield to describe the quadruple-tank process by the following set of non-linear differential equations:

$$
\begin{gathered}
\frac{\mathrm{d} h_{1}}{\mathrm{~d} t}=-\frac{a_{1}}{A_{1}} \sqrt{2 g h_{1}}+\frac{a_{3}}{A_{1}} \sqrt{2 g h_{3}}+\frac{\gamma_{1} k_{1}}{A_{1}} v_{1} \\
\frac{\mathrm{d} h_{2}}{\mathrm{~d} t}=-\frac{a_{2}}{A_{2}} \sqrt{2 g h_{2}}+\frac{a_{4}}{A_{2}} \sqrt{2 g h_{4}}+\frac{\gamma_{2} k_{2}}{A_{2}} v_{2} \\
\frac{\mathrm{d} h_{3}}{\mathrm{~d} t}=-\frac{a_{3}}{A_{3}} \sqrt{2 g h_{3}}+\frac{\left(1-\gamma_{2}\right) k_{2}}{A_{3}} v_{2} \\
\frac{\mathrm{d} h_{4}}{\mathrm{~d} t}=-\frac{a_{4}}{A_{4}} \sqrt{2 g h_{4}}+\frac{\left(1-\gamma_{1}\right) k_{1}}{A_{4}} v_{1}
\end{gathered}
$$

where $A_{i}$ is the cross-section of Tank $i, a_{i}$ is the cross-section of the outlet hole and $h_{i}$ is the water level. The voltage applied to Pump $i$ is $v_{i}$ and the corresponding flow is $k_{i} v_{i}$. The acceleration of gravity is denoted $g$. The parameter values of the laboratory process are given by $\left\{A_{1}, A_{3}\right\}=28 \mathrm{~cm}^{2},\left\{A_{2}, A_{4}\right\}=32 \mathrm{~cm}^{2},\left\{a_{1}, a_{3}\right\}=0.071 \mathrm{~cm}^{2},\left\{a_{2}, a_{4}\right\}=0.051 \mathrm{~cm}^{2}, k_{1}=$ $3.235 \mathrm{~cm}^{3} / \mathrm{Vs}, k_{2}=3.320 \mathrm{~cm}^{3} / \mathrm{Vs}, \gamma_{1}=0.5650$ and $\gamma_{2}=0.47$. The height of each tank is $20 \mathrm{~cm}$.

Following the approach proposed by Shamma and Cloutier [6], a discrete LPV model of quadruple-tank process can be obtained

$$
\begin{aligned}
{\left[\begin{array}{c}
h_{1}(k+1)-h_{1}^{\mathrm{eq}}\left(p_{k+1}\right) \\
h_{2}(k+1)-h_{2}^{\mathrm{eq}}\left(p_{k+1}\right)
\end{array}\right]=} & {\left[\begin{array}{cc}
\tilde{\vartheta}_{1}(k) & 0 \\
0 & \tilde{\vartheta}_{2}(k)
\end{array}\right]\left[\begin{array}{l}
h_{1}(k)-h_{1}^{\mathrm{eq}}\left(p_{k}\right) \\
h_{2}(k)-h_{2}^{\mathrm{eq}}\left(p_{k}\right)
\end{array}\right] } \\
& +\left[\begin{array}{cc}
\tilde{\vartheta}_{3}(k) & \tilde{\vartheta}_{4}(k) \\
\tilde{\vartheta}_{5}(k) & \tilde{\vartheta}_{6}(k)
\end{array}\right]\left[\begin{array}{c}
v_{1}(k)-v_{1}^{\mathrm{eq}}\left(p_{k}\right) \\
v_{2}(k)-v_{2}^{\mathrm{eq}}\left(p_{k}\right)
\end{array}\right]
\end{aligned}
$$

where the scheduling parameters are

$$
\begin{gathered}
\tilde{\vartheta}_{1}(k)=a_{11}\left(p_{k}\right) T_{\mathrm{s}}+1, \quad \tilde{\vartheta}_{2}(k)=a_{22}\left(p_{k}\right) T_{\mathrm{s}}+1, \quad \tilde{\vartheta}_{3}(k)=\left(b_{11}-\frac{\partial h_{1}^{\mathrm{eq}}\left(p_{k}\right)}{\partial h_{4}} b_{41}\right) T_{\mathrm{s}} \\
\tilde{\vartheta}_{4}(k)=-\frac{\partial h_{1}^{\mathrm{eq}}\left(p_{k}\right)}{\partial h_{3}} b_{32} T_{\mathrm{s}}, \quad \tilde{\vartheta}_{5}(k)=-\frac{\partial h_{2}^{\mathrm{eq}}\left(p_{k}\right)}{\partial h_{4}} b_{41} T_{\mathrm{s}}, \quad \tilde{\vartheta}_{6}(k)=\left(b_{22}-\frac{\partial h_{2}^{\mathrm{eq}}\left(p_{k}\right)}{\partial h_{3}} b_{32}\right) T_{\mathrm{s}}
\end{gathered}
$$

and the variables of the scheduling parameters are

$$
\begin{gathered}
a_{11}\left(p_{k}\right)=-\frac{a_{1} \sqrt{(2 g)}}{A_{1} \sqrt{h_{1}(k)}}, \quad a_{22}\left(p_{k}\right)=-\frac{a_{2} \sqrt{2 g}}{A_{2} \sqrt{h_{2}(k)}}, \quad b_{11}=\frac{\gamma_{1} k_{1}}{A_{1}}, \quad b_{22}=\frac{\gamma_{2} k_{2}}{A_{2}} \\
b_{32}=\frac{\left(1-\gamma_{2}\right) k_{2}}{A_{3}}, \quad b_{41}=\frac{\left(1-\gamma_{1}\right) k_{1}}{A_{4}}
\end{gathered}
$$




$$
\begin{aligned}
& h_{1}(k)=\frac{2 \gamma_{1} k_{1} a_{3} v_{1}(k) \sqrt{2 g h_{3}(k)}+\gamma_{1}^{2} k_{1}^{2} v_{1}(k)^{2}+2 a_{3}^{2} g h_{3}(k)}{2 a_{1}^{2} g} \\
& h_{2}(k)=\frac{2 \gamma_{2} k_{2} a_{4} v_{2}(k) \sqrt{2 g h_{4}(k)}+\gamma_{2}^{2} k_{2}^{2} v_{2}(k)^{2}+2 a_{4}^{2} g h_{4}(k)}{2 a_{4}^{2} g}
\end{aligned}
$$

where $h_{i}(k)>1 \mathrm{~cm}$ for $i=1, \ldots, 4$.

Finally, the equilibrium point of (58) is given by:

$$
\begin{aligned}
& h_{1}^{\mathrm{eq}}\left(p_{k}\right)=\frac{2 \gamma_{1} k_{1} a_{3} v_{1}(k) \sqrt{2 g h_{3}(k)}+\gamma_{1}^{2} k_{1}^{2} v_{1}(k)^{2}+2 a_{3}^{2} g h_{3}(k)}{2 a_{1}^{2} g}, \quad v_{1}^{\mathrm{eq}}\left(p_{k}\right)=\frac{a_{3} \sqrt{2 g h_{3}(k)}}{\left(1-\gamma_{2}\right) k_{2}} \\
& h_{2}^{\mathrm{eq}}\left(p_{k}\right)=\frac{2 \gamma_{2} k_{2} a_{4} v_{2}(k) \sqrt{2 g h_{4}(k)}+\gamma_{2}^{2} k_{2}^{2} v_{2}(k)^{2}+2 a_{4}^{2} g h_{4}(k)}{2 a_{4}^{2} g}, \quad v_{2}^{\mathrm{eq}}\left(p_{k}\right)=\frac{a_{4} \sqrt{2 g h_{4}(k)}}{\left(1-\gamma_{1}\right) k_{1}}
\end{aligned}
$$

\section{APPENDIX C: LPV MODEL OF TWIN-ROTOR MIMO SYSTEM}

The dynamic model of this system is proposed by Rahideh and Shaheed [25]:

$$
\begin{aligned}
& \frac{\mathrm{d} i_{a h}}{\mathrm{~d} t}=-\frac{R_{a h}}{L_{a h}} i_{a h}-\frac{k_{a h} \varphi_{h}}{L_{a h}} \omega_{t}+\frac{k_{1}}{L_{a h}} u_{\mathrm{t}} \\
& \frac{\mathrm{d} \omega_{h}}{\mathrm{~d} t}=\frac{k_{a h} \varphi_{h}}{J_{t r}} i_{a h}-\frac{B_{t r}}{J_{t r}} \omega_{t}-\frac{f_{1}\left(\omega_{h}\right) \omega_{h}^{2}}{J_{t r}} \\
& \frac{\mathrm{d} \Omega_{h}}{\mathrm{~d} t}=\frac{l_{t} f_{1}\left(\omega_{h}\right) \omega_{h}^{2} \cos ^{2} \theta_{v}-k_{o h} \Omega_{h}-f_{3}\left(\theta_{h}\right) \theta_{h}}{D \cos ^{2} \theta_{v}+E \sin ^{2} \theta_{v}+F} \\
& +\frac{k_{m} \omega_{v} \sin \theta_{v} \Omega_{v}\left((D-2 E) \cos ^{2} \theta_{v}-E \sin ^{2} \theta_{v}-F\right)}{\left(D \cos ^{2} \theta_{v}+E \sin ^{2} \theta_{v}+F\right)^{2}} \\
& +\frac{k_{m} \cos \theta_{v}\left(k_{a v} \varphi_{v} i_{a v}-B_{m r} \omega_{v}-f_{4}\left(\omega_{v}\right) \omega_{v}^{2}\right)}{\left(D \cos ^{2} \theta_{v}+E \sin ^{2} \theta_{v}+F\right) J_{m r}} \\
& \frac{\mathrm{d} \theta_{h}}{\mathrm{~d} t}=\Omega_{h} \\
& \frac{\mathrm{d} i_{a v}}{\mathrm{~d} t}=-\frac{R_{a v}}{L_{a v}} i_{a v}-\frac{k_{a v} \varphi_{v}}{L_{a v}} \omega_{v}+\frac{k_{2}}{L_{a v}} u_{\mathrm{m}} \\
& \frac{\mathrm{d} \omega_{v}}{\mathrm{~d} t}=\frac{k_{a v} \varphi_{v}}{J_{m r}} i_{a v}-\frac{B_{m r}}{J_{m r}} \omega_{v}-\frac{f_{4}\left(\omega_{v}\right) \omega_{v}^{2}}{J_{m r}} \\
& \frac{\mathrm{d} \Omega_{v}}{\mathrm{~d} t}=\frac{l_{m} f_{5}\left(\omega_{v}\right) \omega_{v}^{2}+k_{g} \Omega_{h} f_{5}\left(\omega_{v}\right) \omega_{v}^{2} \cos \theta_{v}-k_{o v} \Omega_{v}}{J_{v}} \\
& +\frac{g\left((A-B) \cos \theta_{v}-C \sin \theta_{v}\right)-0.5 \Omega_{v}{ }^{2} H \sin \left(2 \theta_{v}\right)}{J_{v}} \\
& +\frac{k_{t}\left(k_{a h} \varphi_{h} i_{a h}-B_{t r} \omega_{h}-f_{1}\left(\omega_{h}\right) \omega_{h}^{2}\right)}{J_{v} J_{t r}} \\
& \frac{\mathrm{d} \theta_{v}}{\mathrm{~d} t}=\Omega_{v}
\end{aligned}
$$


The functions and constants of TRMS model can be found in [25]. Following the approach proposed by Kwiatkowski et al. [46], a discrete-time LPV model of the TRMS can be obtained by considering as scheduling variables the available measurements outputs: azimuth angle of the beam $\theta_{h}(k)$ and pitch angle of the beam $\theta_{v}(k)$

$$
\begin{aligned}
{\left[\begin{array}{c}
i_{a h}(k+1) \\
\omega_{h}(k+1) \\
\Omega_{h}(k+1) \\
\theta_{h}(k+1) \\
i_{a v}(k+1) \\
\omega_{v}(k+1) \\
\Omega_{v}(k+1) \\
\theta_{v}(k+1)
\end{array}\right]=} & {\left[\begin{array}{cccccccc}
a_{11} & a_{12} & 0 & 0 & 0 & 0 & 0 & 0 \\
a_{21} & \tilde{\vartheta}_{1}(k) & 0 & 0 & 0 & 0 & 0 & 0 \\
0 & \tilde{\vartheta}_{2}(k) & \tilde{\vartheta}_{3}(k) & \tilde{\vartheta}_{4}(k) & \tilde{\vartheta}_{5}(k) & \tilde{\vartheta}_{6}(k) & \tilde{\vartheta}_{7}(k) & 0 \\
0 & 0 & a_{43} & 0 & 0 & 0 & 0 & 0 \\
0 & 0 & 0 & 0 & a_{55} & a_{56} & 0 & 0 \\
0 & 0 & 0 & 0 & a_{65} & \tilde{\vartheta}_{8}(k) & 0 & 0 \\
a_{71} & \tilde{\vartheta}_{9}(k) & \tilde{\vartheta}_{10}(k) & 0 & 0 & \tilde{\vartheta}_{11}(k) & a_{77} & \tilde{\vartheta}_{12}(k) \\
0 & 0 & 0 & 0 & 0 & 0 & a_{87} & 0
\end{array}\right]\left[\begin{array}{c}
i_{a h}(k) \\
\omega_{h}(k) \\
\Omega_{h}(k) \\
\theta_{h}(k) \\
i_{a v}(k) \\
\omega_{v}(k) \\
\Omega_{v}(k) \\
\theta_{v}(k)
\end{array}\right] } \\
& +\left[\begin{array}{cc}
b_{11} & 0 \\
0 & 0 \\
0 & 0 \\
0 & 0 \\
0 & b_{52} \\
0 & 0 \\
0 & 0 \\
0 & 0
\end{array}\right]\left[\begin{array}{c}
v_{1}(k) \\
v_{2}(k)
\end{array}\right]
\end{aligned}
$$

where

$$
\begin{gathered}
a_{11}=-\frac{R_{a h}}{L_{a h}}, \quad a_{12}=-\frac{k_{a h} \varphi_{h}}{L_{a h}}, \quad a_{21}=\frac{k_{a h} \varphi_{h}}{J_{t r}}, \quad a_{43}=1, \quad a_{55}=-\frac{R_{a v}}{L_{a v}}, \quad a_{56}=-\frac{k_{a v} \varphi_{v}}{L_{a v}} \\
a_{65}=\frac{k_{a v} \varphi_{v}}{J_{m r}}, \quad a_{71}=\frac{k_{t} k_{a v}}{J_{v} J_{t r}}, \quad a_{77}=-\frac{k_{o v}}{J_{v}}, \quad a_{87}=1, \quad b_{11}=\frac{k_{1}}{L_{a h}}, \quad b_{52}=\frac{k_{2}}{L_{a h}}
\end{gathered}
$$

and the LPV parameters in function of the scheduling variables are

$$
\begin{gathered}
\tilde{\vartheta}_{1}(k)=\frac{-B_{t r}-f_{1}\left(\omega_{h}\right) \omega_{h}}{J_{t r}}, \quad \tilde{\vartheta}_{2}(k)=\frac{l_{t} f_{2}\left(\omega_{h}\right) \omega_{h} \cos \theta_{v}}{D \cos ^{2} \theta_{v}+E \sin ^{2} \theta_{v}+F} \\
\tilde{\vartheta}_{3}(k)=-\frac{k_{o h}}{D \cos ^{2} \theta_{v}+E \sin ^{2} \theta_{v}+F}, \quad \tilde{\vartheta}_{4}(k)=-\frac{f_{3}\left(\theta_{h}\right) \theta_{h}}{D \cos ^{2} \theta_{v}+E \sin ^{2} \theta_{v}+F} \\
\tilde{\vartheta}_{5}(k)=\frac{k_{m} \cos \theta_{v} k_{a}}{\left(D \cos ^{2} \theta_{v}+E \sin ^{2} \theta_{v}+F\right) J_{m r}}, \quad \tilde{\vartheta}_{6}(k)=-\frac{k_{m} \cos \theta_{v}\left(B_{m r}+f_{4}\left(\omega_{v}\right) \omega_{v}\right)}{\left(D \cos ^{2} \theta_{v}+E \sin ^{2} \theta_{v}+F\right) J_{m r}} \\
\tilde{\vartheta}_{7}(k)=\frac{k_{m} \omega_{v} \sin \theta_{v}\left(D \cos ^{2} \theta_{v}-E \sin ^{2} \theta_{v}-F-2 \cos ^{2} \theta_{v} E\right)}{\left(D \cos ^{2} \theta_{v}+E \sin ^{2} \theta_{v}+F\right)^{2}}, \quad \tilde{\vartheta}_{8}(k)=\frac{-B_{m r}-f_{4}\left(\omega_{v}\right) \omega_{v}}{J_{m r}} \\
\tilde{\vartheta}_{9}(k)=\frac{k_{t}\left(-B_{t r}-f_{1}\left(\omega_{h}\right) \omega_{h}\right.}{J_{v} J_{t r}}, \quad \tilde{\vartheta}_{10}(k)=\frac{-0.5 \Omega_{h} H \sin \left(2 \theta_{v}\right)+k_{g} f_{5}\left(\omega_{v}\right) \cos \theta_{v}}{J_{v}} \\
\tilde{\vartheta}_{11}(k)=\frac{l_{m} f_{5}\left(\omega_{v}\right) \omega_{v}}{J_{v}}, \quad \tilde{\vartheta}_{12}(k)=\frac{g\left((A-B) \cos \theta_{v}-C \sin \theta_{v}\right)}{J_{v} \theta_{v}}
\end{gathered}
$$


for $\omega_{h}>0, \omega_{v}>0$. In case that the values of $\theta_{v}$ are in $-0.001<\theta_{v}<0$, the value is approximated as $\theta_{v}=-0.001$ and if $0<\theta_{v}<0.001$, the value is approximated as $\theta_{v}=0.001$. Notice that the LPV parameters depend additionally to the measured scheduled variables $\left(\theta_{v}\right.$ and $\left.\theta_{h}\right)$ from the following state variables: $\omega_{h}, \omega_{v}$ and $\Omega_{h}$. These state variables as well as their interval of uncertainty are estimated from the interval LPV observer.

\section{ACKNOWLEDGEMENTS}

This work has been partially funded by the grant CICYT HYFA DPI2008-01996 and WATMAN DPI200913744 of Spanish Ministry of Education and by a grant from Consejo Nacional de Ciencia y Tecnologia de México (CONACyT).

\section{REFERENCES}

1. Chen J, Patton RJ. Robust Model-based Fault Diagnosis for Dynamic Systems. Kluwer Academic Publishers: Dordrecht, 1999.

2. Gertler J. Fault Detection and Diagnosis in Engineering Systems. Marcel Dekker: New York, 1998.

3. Patton RJ, Frank PM, Clark RN. Issues of Fault Diagnosis for Dynamic Systems. Springer: Berlin, Heidelberg, 2000.

4. Bokor J. Fault detection and isolation in nonlinear systems. The Seventh IFAC Symposium on Fault Detection, Supervision and Safety of Technical Processes (Plenary Session) 2009, Barcelona, Spain, 2009.

5. Apkarian P, Gahinet P, Becker G. Self-scheduled $H_{\infty}$ control of linear parameter-varying systems: a design example. Automatica 1995; 31(9):1251-1261.

6. Shamma JS, Cloutier JR. Gain scheduled missile autopilot design using linear parameter varying transformations. AIAA Journal of Guidance, Control, and Dynamics 1993; 16(2):256-263.

7. Andrés M, Balas GJ. Development of linear-parameter-varying models for aircraft. Journal of Guidance, Control and Dynamics 2004; 27(2):218-228.

8. Chen J, Patton RJ, Zhang HY. Design of unknown input observers and robust fault-detection filters. International Journal of Control 1996; 63(1):85-105.

9. Patton RJ, Liu GP. Robust control design via eigenstructure assignment, genetic algorithms and gradient-based optimization. IEE Proceedings Control Theory and Application 1994; 141(3):202-208.

10. Bokor J, Balas G. Detection filter design for LPV systems: a geometric approach. Automatica 2004; 40:511-518.

11. Rodrigues M, Theilliol D, Sauter D. Design of an active fault tolerant control and polytopic unknown input observer for systems described by a multi-model representation. The 44th IEEE Conference on Decision and Control and European Control Conference ECC, Sevilla, Spain, 2005.

12. Grenaille S, Henry D, Zolghadri A. A method for designing fault diagnosis filters for LPV polytopic systems. Journal of Control Science and Engineering 2008; 2008:1-11.

13. Armeni S, Casavola A, Mosca E. Robust fault detection and isolation for LPV systems under a sensitivity constraint. International Journal of Adaptive Control and Signal Processing 2009; 23(1):55-72.

14. Horak DT. Failure detection in dynamic systems with modelling errors. Journal of Guidance, Control, and Dynamics 1988; 11(6):508-516.

15. Emami-Naeini A, Akhter MM, Rock SM. Effect of model uncertainty of failure detection: the threshold selector. IEEE Transactions on Automatic Control 1988; 33(12):1106-1115.

16. Fagarasan I, Ploix S, Gentil S. Causal fault detection and isolation based on a set-membership approach. Automatica 2004; 2099-2110.

17. Puig V, Quevedo J, Escobet T, Nejjari F, de las Heras S. Passive robust fault detection of dynamic processes using interval models. IEEE Transactions on Control Systems Technology 2008; 16:1083-1089.

18. Sainz MÁ, Armengol J, Vehí J. Fault detection and isolation of the three-tank system using the modal interval analysis. Journal of Process Control 2002; 12(2):325-338.

19. Ploix S, Adrot O. Parity relations for linear uncertain dynamic systems. Automatica 2006; 42(9):1553-1562.

20. Alamo T, Bravo JM, Camacho EF. Guaranteed state estimation by zonotopes. Automatica 2005; 41(6):1035-1043.

21. Puig V, Stancu A, Escobet T, Nejjari F, Quevedo J, Patton RJ. Passive robust fault detection using interval observers: application to the DAMADICS benchmark problem. Control Engineering Practice 2006; 14(6): 621-633.

22. Gouzé JL, Rapaport A, Hadj-Sadok MZ. Interval observers for uncertain biological systems. Journal of Ecological Modelling 2000; 133:45-56.

23. Johansson KH. The Quadruple-tank process: a multivariable laboratory process with an adjustable zero. IEEE Transactions on Control Systems Technology 2000; 8:456-465.

24. Feedback Instruments Ltd, Crowborough, U.K. Twin Rotor MIMO System Advanced Teaching Manual 1 (33-007-4M5), 1998.

25. Rahideh A, Shaheed MH. Dynamic modelling of a twin rotor MIMO system using grey box approach. Proceedings of the Fifth International Symposium on Mechatronics and its Applications (ISMA 2008), Amman, Jordan, 2008. 
26. Murray-Smith R, Johansen TA. Multiple Model Approaches to Modelling and Control. Taylor \& Francis: London, 1997.

27. Bamieh B, Giarré L. Identification of linear parameter varying models. International Journal Robust Nonlinear Control 2002; 2(12):841-853.

28. Blesa J, Puig V, Bolea Y. Fault detection using interval LPV models in an open-flow canal. Control Engineering and Practice 2010; 18(5):460-470.

29. Meseguer J, Puig V, Escobet T. Observer gain effect in linear interval observer-based fault detection. The Sixth IFAC Symposium on Fault Detection, Supervision and Safety of Technical Processes (SAFEPROCESS), vol. 6, 2006.

30. Heemels WPMH, Daafouz J, Millerioux G. Design of observer-based controllers for LPV systems with unknown parameters. The 48th IEEE Conference on Decision and Control $(C D C)$ and the 28th Chinese Control Conference, Shanghai, China, 2009; 1836-1841.

31. Chilali M, Gahinet P, Apkarian P. Robust pole placement in LMI regions. IEEE Transactions on Automatic Control 1999; 44(12):2257-2270.

32. Puig V, Quevedo J, Escobet T. Robust fault detection approaches using interval models. IFAC World Congress (b’02), Barcelona, Spain, 2002.

33. Maksarov D, Norton JP. State bounding with ellipsoidal set description of the uncertainty. International Journal of Control 1996; 65(5):847-866.

34. Landau ID, Zito G. Digital Control Systems: Design, Identification and Implementation. Springer: Berlin, 2006.

35. Moore RE. Interval Analysis. Prentice-Hall: Englewood Cliffs, NJ, 1966.

36. Combastel C. A state bounding observer for uncertain non-linear continuous-time systems based on Zonotopes. The 44th IEEE Conference on Decision and Control and European Control Conference, CDC-ECC'05, Seville, Spain, 2005.

37. Kuhn W. Rigorously computed orbits of dynamical systems without the wrapping effect. Computing 1998; 61(1):47-67.

38. Combastel C. A state bounding observer based on Zonotopes. European Control Conference, Cambridge, U.K., 2003.

39. Peng Y, Youssouf A, Arte P, Kinnaert M. A complete procedure for residual generation and evaluation with application to a heat exchanger. IEEE Transactions on Control Systems Technology 1997; 5:542-555.

40. Balas GJ. Linear, parameter-varying control and its application to a turbofan engine. International Journal of Robust and Nonlinear Control 2002; 12(9):763-796.

41. Shamma JS. Linearization and gain-scheduling. In The Control Handbook, Levine WS (ed.). CRC Press: Boca Raton, 1996; 388-396.

42. Kwiatkowski A, Werner H. PCA-based parameter set mappings for LPV models with fewer parameters and less overbounding. IEEE Transactions on Control Systems Technology 2008; 6(4):781-788.

43. Kumar A, Anderson MR. A comparison of LPV modeling techniques for aircraft control. AIAA Guidance, Navigation, and Control Conference and Exhibit, Denver. AIAA-2000-4458, 2000.

44. Biannic JM. Commande Robuste des Systèmes à Paramètres Variables. Application en Aéronautique. Ph.D. Thesis, Centre d'Études et de Recherche de Toulouse, Department DERA, Toulouse, France, 1996.

45. Barber CB, Dobkin DP, Huhdanpaa HT. The Quickhull algorithm for convex hulls. ACM Transactions on Mathematical Software 1996; 22(4):469-483.

46. Kwiatkowski A, Boll MT, Werner H. Automated generation and assessment of affine LPV models. Proceedings of the 45th IEEE Conference on Decision and Control, San Diego, CA, U.S.A., 2006; 6690-6695.

47. Montes de Oca S, Puig V. Fault diagnosis of nonlinear systems using LPV interval observers. The 20th International Workshop on Principle of Diagnosis (DX-09), 2009. 